\title{
WestVirginiaUniversity
}

THE RESEARCH REPOSITORY @ WVU

Graduate Theses, Dissertations, and Problem Reports

2012

\section{Analysis and detection of fingerprint creases}

Olaoluwa Peter Laseinde

West Virginia University

Follow this and additional works at: https://researchrepository.wvu.edu/etd

\section{Recommended Citation}

Laseinde, Olaoluwa Peter, "Analysis and detection of fingerprint creases" (2012). Graduate Theses, Dissertations, and Problem Reports. 434.

https://researchrepository.wvu.edu/etd/434

This Thesis is protected by copyright and/or related rights. It has been brought to you by the The Research Repository @ WVU with permission from the rights-holder(s). You are free to use this Thesis in any way that is permitted by the copyright and related rights legislation that applies to your use. For other uses you must obtain permission from the rights-holder(s) directly, unless additional rights are indicated by a Creative Commons license in the record and/ or on the work itself. This Thesis has been accepted for inclusion in WVU Graduate Theses, Dissertations, and Problem Reports collection by an authorized administrator of The Research Repository @ WVU. For more information, please contact researchrepository@mail.wvu.edu. 


\title{
Analysis and detection of fingerprint creases
}

\author{
by \\ Olaoluwa Peter Laseinde \\ Thesis submitted to the \\ Benjamin M. Statler College of Engineering and Mineral Resources \\ at West Virginia University \\ in partial fulfillment of the requirements \\ for the degree of \\ Master of Science \\ in \\ Computer Science \\ Donald Adjeroh, Ph.D. \\ Katerina Goseva-Popstojanova, Ph.D. \\ Arun A. Ross, Ph.D., Chair \\ Lane Department of Computer Science and Electrical Engineering \\ Morgantown, West Virginia \\ 2012
}

Keywords: Fingerprint recognition, creases, residue estimation, phase unwrapping minutiae, Gabor filter, Hough transform.

Copyright 2012 Olaoluwa Peter Laseinde 


\author{
Abstract \\ Analysis and detection of fingerprint creases \\ Olaoluwa Peter Laseinde \\ Master of Science in Computer Science \\ West Virginia University \\ Arun A. Ross, Ph.D., Chair
}

Fingerprint is a biometric trait that is widely used for human identification and verification. Most fingerprint biometric systems make use of certain salient features on the fingerprint, including minutiae points, pores, and singular points, for comparing two fingerprint images. In this work, we explore the possibility of using fingerprint creases for comparing two fingerprints. Creases can be described as white lines or scars on a fingerprint image. Recent studies have determined that some creases are genetically influenced although the origin of creases has not been completely characterized. While no published work exists for crease matching, some studies have explored the problem of automated crease detection. In this thesis, we study the possibility of using creases for fingerprint matching. We also suggest two techniques to automatically extract creases from an input fingerprint image. Finally, we study the correlation between fingerprint creases and age of an individual. 


\section{Acknowledgements}

I will like to express my gratitude to my supervisor Dr Arun Ross who accepted to supervise my research and also engaged me in other research. He has really been a source of encouragement and motivation throughout the period of my masters, his intelligent ideas has helped me tremendously and I would not have achieved this much if not for his support and understanding. I have also learnt through him the definition of relentless and doggedness in achieving set goals. His exceptional teaching ability has made him stand out and I also learnt a lot in the few classes of his that I took.

I would also like to thank Dr Adjeroh and Dr Katerina Goseva-Popstojanova who accepted to be on my committee, Dr Adjeroh has been of tremendous encouragement to me especially when I needed counsel on my courses and research. I am greatly thankful to Dr Katerina who made herself available for discussions on my research and was always willing to provide advise on my work, she is also an outstanding teacher.

My sincere appreciation goes to my lab colleagues: Asem helped with some of my programming codes in this thesis; Raghavender and Eric helped in reviewing my thesis and they were always willing to help in any way; Ravi, Raghunandan, Emanuela, Gizem, Chen were highly supportive.

I am indebted to my family and friends including Muniru, Tokunbo who were highly supportive .

Finally I thank the all sovereign God for being my source of strength and giving me life and good health to go through this academic journey. 


\section{Contents}

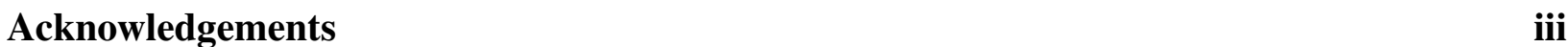

List of Figures

List of Tables $\quad$ viii

1 Introduction $\quad 1$

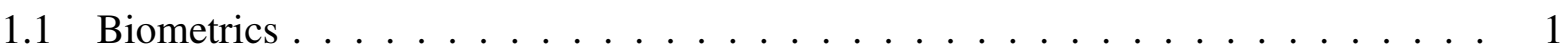

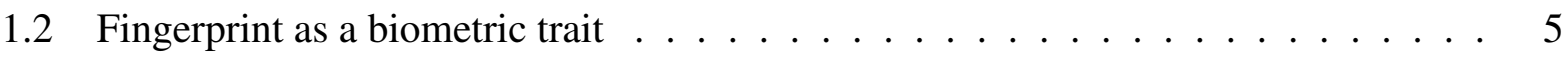

1.3 Fingerprint characteristic . . . . . . . . . . . . . . . . . 9

1.3.1 Fingerprint creases and importance . . . . . . . . . . . . . . 9

1.4 Problem Statement and Contributions _ . . . . . . . . . . . . . . . . 12

2 Related work $\quad 15$

2.1 Introduction . . . . . . . . . . . . . . . . . . . 15

2.2 Hough transform for detecting lines in objects . . . . . . . . . . . . . 16

2.3 Crease representation as parameterized rectangle $\ldots \ldots \ldots \ldots$

3 Matching and Classification $\quad 22$

3.1 Fingerprint matching based on creases . . . . . . . . . . . . . . 22

3.1 .1 Introduction . . . . . . . . . . . . . . . . . 22

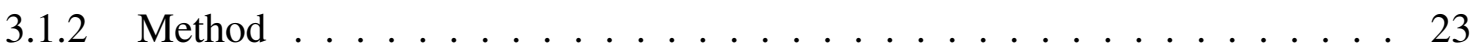

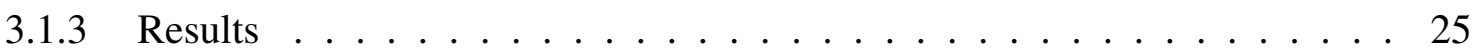

3.2 Classification of age groups based on creases $\ldots \ldots \ldots \ldots$

3.2 .1 Introduction . . . . . . . . . . . . . . . . . 30

3.2 .2 Method . . . . . . . . . . . . . . . . 30

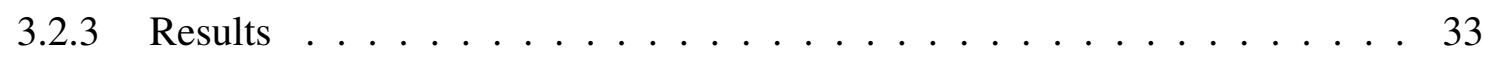

4 Automated fingerprint crease detection techniques $\quad 37$

4.1 Pixel intensity based method . . . . . . . . . . . . . . . . . . 37

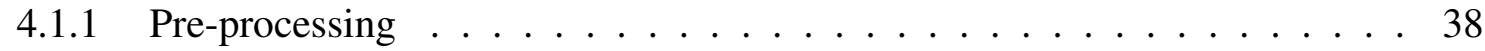

4.1 .2 Obtaining the Region Of Interest (ROI) $\ldots \ldots \ldots \ldots$. . . . . . . 40

4.1 .3 Gaussian Filtering . . . . . . . . . . . . . . . . 43

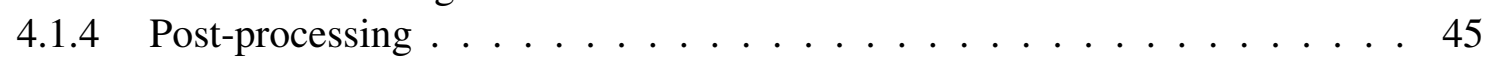

4.2 Local ridge orientation field method . . . . . . . . . . . . . . . . 47 
4.2.1 Preprocessing/enhancement . . . . . . . . . . . . . . . 47

4.2.2 Ridge orientation estimation . . . . . . . . . . . . . . . . 48

4.2 .3 Region of interest estimate (ROI) . . . . . . . . . . . . . . 51

4.2 .4 Residue Estimation . . . . . . . . . . . . . . . . . . 51

4.2 .5 Post Processing . . . . . . . . . . . . . . . . . 52

4.3 Validation of automated crease detection algorithm . . . . . . . . . . . 54

5 Conclusion $\quad 60$

$\begin{array}{ll}\text { References } & 62\end{array}$ 


\section{List of Figures}

1.1 Different biometric traits . . . . . . . . . . . . . . . . 3

1.2 Typical biometric recognition process . . . . . . . . . . . . . 4

1.3 A fingerprint displaying the physical structure . . . . . . . . . . . 6

1.4 Effect of distortion on minutiae location. Red points are ridge endings while green points are mostly ridge bifurcations $\ldots \ldots \ldots \ldots$. . . . . . . . 8

1.5 An enhanced fingerprint: (a) whorl, 2 deltas, 2 cores (b) loop, 1 delta, 1 core, (c) $\operatorname{arch}($ no delta or core) . . . . . . . . . . . . . . . . . . . . 10

1.6 Different manifestations of creases (a) Good quality Fingerprint with obvious creases, (b) Fingerprint with less obvious creases appearing as scattered lines. . . . . . . . 11

1.7 A typical fingerprint layer structure showing the fingerprint layer (dermis) and

1.8 False minutiae and removal: (a) Original image, (b) Thinned image, (c) Thinned image with both spurious and real minutiae, (d) Original image with superimposed real/actual minutiae. . . . . . . . . . . . . . . . . . . . 13

2.1 Angular difference between the fingerprint ridges and creases. . . . . . . . . . . 16

2.2 (a) Image space representation, (b) An example of Hough space representation showing the points on the space with the peak values highlighted. . . . . . . . 17

2.3 Application of Hough transform on fingerprint images[15] (a) Original image, (b) Hough transform Space (c) Line detected after applying Hough transform. . . . . . 18

2.4 (a) A fingerprint with a crease structure. (b) A crease represented by a rectangle (c) A parameterized triangle in the $\mathrm{x}$-y domain. . . . . . . . . . . . . 18

2.5 (a) Fingerprint creases (b) Creases showing the detailed width representation of ridge and valley as being the same. . . . . . . . . . . . . 20

2.6 Output of crease detection algorithm obtained from [11] . . . . . . . . . . . 21

3.1 An example of manually marked crease . . . . . . . . . . . . . . . 23

3.2 ROC curve for FAR and FRR . . . . . . . . . . . . . . . . . . . . 25

3.3 Distribution of normalized genuine and impostor scores for the crease matching

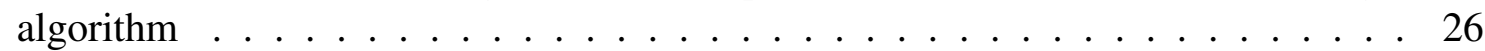

3.4 ROC curve for FAR and FRR . . . . . . . . . . . . . . . . . 27

3.5 Distribution of normalized genuine and impostor scores for the crease matching



3.6 Distribution of number of creases found on fingerprint $\ldots \ldots \ldots$ 
3.7 ROC Curve based on creases less than or equal $3 \ldots \ldots \ldots$

3.8 ROC Curve based on creases greater than $3 \ldots \ldots$. . . . . . . . . . . . . . . 29

3.9 Images showing the regions covered by creases manually marked out. . . . . . . . 31

3.10 Histogram showing left thumb and left index fingers (ages 0-20, 50-80) . . . . . . 32

3.11 Histogram showing left thumb and left index finger (ages $0-30,50-80$ ) . . . . . . 32

3.12 Histogram showing left thumb and left index finger (ages 0-40, 50-80) . . . . . . . 33

3.13 ANOVA analysis of left index fingers (overlapping age groups) . . . . . . . . . 35

3.14 ANOVA analysis of left thumb fingers (overlapping age groups) $\ldots \ldots \ldots$

3.15 ANOVA analysis of left index fingers (distinct age groups) . . . . . . . . . . . 35

3.16 ANOVA analysis of left thumb fingers (distinct age groups) . . . . . . . . . . . 36

4.1 Flowchart of the pixel intensity based algorithm to detect creases. . . . . . . . . . 38

4.2 Effects of various preprocessing method: (a) Original image (b) image obtained after applying normalization (c) image obtained after applying histogram equalization.

4.3 ROI generated using 2 different techniques (a) Original Image, (b) ROI with block size 16, (c) ROI with block size 8, (d) ROI with block size 2, (e) ROI using the second method. . . . . . . . . . . . . . . . . . . . . 42

4.4 A mask superimposed on the enhanced image . . . . . . . . . . . . . . . . 43

4.5 Different line mask filters . . . . . . . . . . . . . . . . . . . . . . 43

4.6 (a) Superimposed mask on the enhanced image (b) Image after applying Gaussian filter . . . . . . . . . . . . . . . . . . . . 44

4.7 (a) Image after applying Gaussian filter, and (b) after thresholding . . . . . . . . . 45

4.8 Sample output obtained after post-processing . . . . . . . . . . . . . . . 46

4.9 Schematic for crease detection based on ridge orientation field. . . . . . . . . . . . 47

4.10 Orientation field (gradient based) superimposed on a fingerprint. . . . . . . . . . . 49

4.11 Effect of image quality on ridge orientation field (1) Good quality fingerprint (2)

Poor quality fingerprint. . . . . . . . . . . . . . . . . . 50

4.12 Ridge orientation field of a fingerprint showing regions containing residue points . 52

4.13 Fingerprint with the creases after applying Euclidean distance at a given threshold. 53

4.14 Results obtained using $\operatorname{method}(1) \ldots \ldots \ldots \ldots$. . . . . . . . . . . 54

4.15 Performance of crease detection on a fingerprint image using method(2). . . . . . . 55

4.16 Graph displaying performance of $\operatorname{method}(1) \ldots \ldots \ldots$. . . . . . . . . . . 58

4.17 Graph displaying performance of $\operatorname{method}(2) . \ldots \ldots$. . . . . . . . . . 59 


\section{List of Tables}

4.1 Data describing the percentage of crease detected based on method(1) . . . . . . 56

4.2 Performance analysis from method(1) based on pixel intensity . . . . . . . . . 57

4.3 Performance analysis from method(2) based on residue points . . . . . . . . . 58 


\section{Chapter 1}

\section{Introduction}

\subsection{Biometrics}

Biometrics involves analyzing biological data which may be in the form of measurements of human body characteristics such as fingerprint, iris, retina, DNA, etc. with the main goal of recognizing people based on such characteristics. It can also be defined as the science and technology of automatically recognizing users based on some unique and universal behavioral or physical characteristics. Recognition of people can be classified into two categories, identification and verification.

Identification of a user occur when a user submits his biometric data. The data is checked against all enrolled data in the database to confirm if there is a match. Identification therefore involves comparing one input sample with multiple stored samples and is sometimes referred to as one-to-many comparisons. Verification occurs when a user submits his biometric data and also provides a unique label such as name, social security number, or passport number. The system attempts to compare the newly collected data (query) with an already stored data (template) based on the label supplied. If there exists a match the user is said to be a genuine user otherwise the user is considered an impostor by the system. Verification involves a one-to-one comparison or one-to-one matching. Hence the process is much faster than identification.

Because matching is based on measurements taken from human body, it is not always possible to obtain the exact measurement each time a new sample is collected. Matching may therefore not be $100 \%$ accurate in biometric systems and hence some possible errors could occur. Such errors 
can be described as false accepts and false rejects. False accept occurs when an impostor pretends to be a genuine user and the biometric system incorrectly confirms him/her to be genuine, while false reject occurs when a genuine user is misclassified as an impostor by the system. The occurrence of such errors should be minimized as much as possible. In applications such as an ATM, it would be desirous to have a very low false accept rate while in criminal investigations it would be more desirous to have a very low false reject rate so that a criminal is not missed by the biometric system.

Biometric data are stored in a database in form of biometric templates. It should be noted that in some systems only the feature is stored and not the raw biometric data. An example is a fingerprint recognition system based on minutiae points. Minutiae points are stored in the database as templates. Minutiae points template can be described as a two-dimensional table containing the geometric locations of the points where the minutiae are located on the raw image and their respective directions.

The terms biometrics and biometry date back to the early 20th century [3] where the concept was used in biological science to refer to the application of mathematical and statistical methods for data analysis. Biometrics has been found to be very useful long before the advent of highly sophisticated computers that are present today. Fingerprint is the most widely used trait due to its wide success in numerous applications [3]. Other biometric traits include iris, retina, vein pattern, hand geometry, face, keystroke dynamics, signature, gait, and voice (Figure 1.1). 


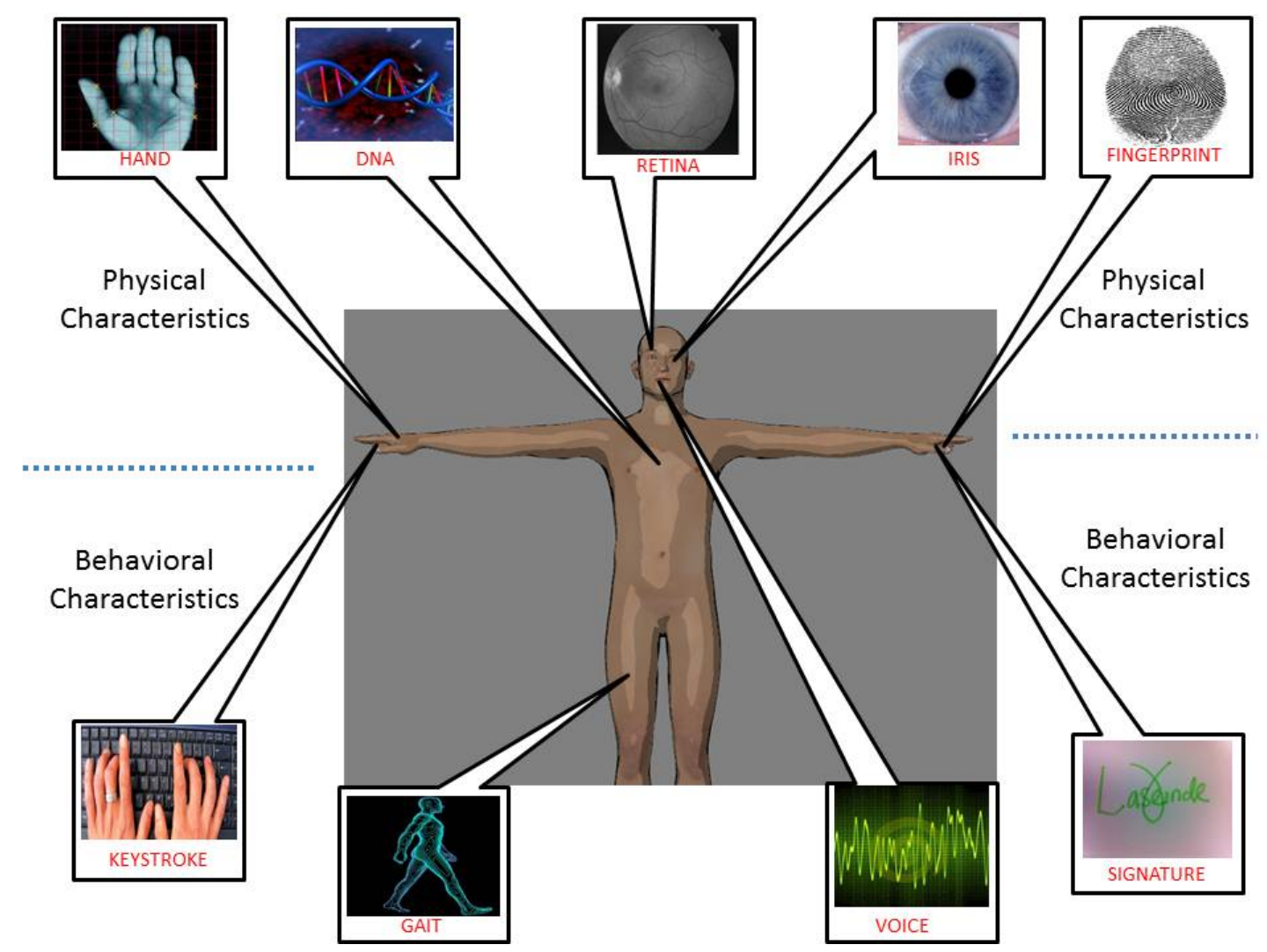

Figure 1.1: Different biometric traits

Traits are classified into two categories, namely, behavioral and physical characteristics. Keystroke dynamics, signature, voice and gait can be classified as behavioral patterns because they are learnt by the user over a time period. Iris, face and fingerprint can be classified as physical patterns since they are directly related to anatomical attributes of an individual.

A typical biometric process involves four major modules: sensor module, feature extraction module, matcher module and decision making module [1].

- Sensor module involves capturing raw biometric data which can be in form of fingerprint images, voice signals, etc. using devices like fingerprint sensors and microphone. The raw biometric data is sometimes referred to as a sample. Some other components such as keyboard and screen may be involved at this stage to capture non biometric information of the user such as entering the label information of users via the keyboard [5]. In the case of fingerprint, which is the subject of this thesis, capturing images was previously done by applying ink on the subject's fingertip, and subjects were requested to make prints by rolling 


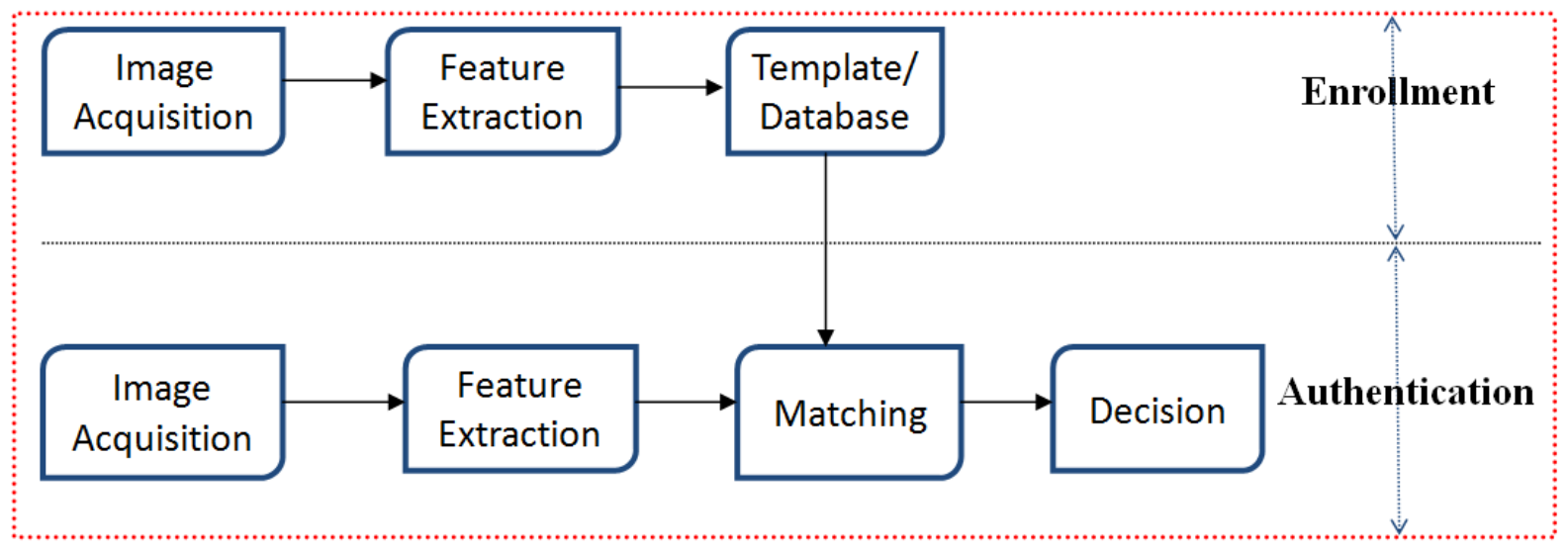

Figure 1.2: Typical biometric recognition process

the finger or pressing hard against a paper card. The imprinted fingerprint was then imaged using a digital camera and this was usually captured at a resolution of 500 dpi [5]. Because direct contact with the sensor is not required, it has been widely used in collecting latent fingerprint from crime scenes through the use of chemical processes to highlight the print [5]. Recently, more robust applications have led to live-scan sensing of fingerprint which may or may not require user to have direct contact with the sensor. Some of these sensors include optical sensors, solid state sensors and ultra sound sensors [5]. Some errors associated with the sensor module may include failure to detect and failure to capture. Failure to detect occurs when a user presents his fingerprint but the sensor fails to detect the fingerprint. Failure to capture occurs when the sensor actually detects the fingerprint but fails to capture a reasonable image. This may be due to bad positioning of the finger.

- Feature extraction module involves the extraction of salient features from the raw biometric data obtained through the sensor by using a feature extractor. In the case of fingerprints, features could include minutiae points, pores, and singular points. In hand geometry, width, thickness, length of fingers, etc. are possible features that can be collected. The output of this module is usually in an expressive compact form known as feature set.

This stage also presents some errors including failure to process; this is usually due to the poor quality fingerprint and the algorithm may be unable to extract useful information [5]. Very similar to failure to process is the failure to enroll. It is the error reported when the 
image is of poor quality and the salient feature extracted is not good enough to be enrolled in a system.

- Matcher module involves making a comparison of the acquired biometric data obtained from the feature extraction module against the set of stored features in the database in order to establish a match or non-match. These modules usually require a form of pre-selection or filtering process which involves reducing the database size to be checked in order to reduce computation and time. Following the pre-selection comes the score generation based on comparison. In identification, the scores are ordered and ranked, a threshold is also applied to establish similarity or dissimilarity.

- Decision making module determines if the claimed identity of the user is accepted or rejected in a verification system. Some of the errors associated with this stage includes false match and false non-match. False match is the error that occurs when the system declares an impostor as a genuine user while the false non-match is the error that occurs when the system declare that a genuine user is an impostor and the user is denied access.

The objective of a good biometric system is to make sure that the error rate is minimized as much as possible. It has been confirmed that traits such as the iris have low false accept rates [6].

\subsection{Fingerprint as a biometric trait}

Among the various biometric traits, fingerprint has been studied for a long time and is widely accepted due to many attractive characteristics including universality, uniqueness and acceptability. Fingerprint is made up of the interleaved ridges and valleys sometimes referred to as furrows and these features are found at the tip of the fingers. The first scientific paper on fingerprint furrow and pores was done by Nehemiah Grew in 1864 while in 1823 Purkinje proposed the first classification of fingerprints into nine different categories [5].

A standard classification that is well known was carried out by Edward Henry in 1899, and is often referred to as the Henry's system. Fingerprint was applied in forensics in the early 20th century and this led to the formation of a lot of fingerprint agencies worldwide. An example is the FBI facility that was set up in 1924. 




Figure 1.3: A fingerprint displaying the physical structure

Ridges and valleys are formed on the finger around the first seven months of fetal development leaving a permanent set of features which is highly unique and can easily be distinguished by using a fingerprint scanner. The formation of fingerprint has been observed to be genetically controlled. Timing factor of the appearance or the disappearance of the volar pads determines the nature of fingerprints [7].

Glenn Langenburg [8] explained by stating that the primary ridges, which are usually the first to appear on the volar pads, appear at the tip of the finger, and if this appearance is observed when the volar pads are still very visible, then it is likely to be a whorl. He also claimed that a loop is formed when the volar pads are less visible and withdrawn. The arch pattern is formed when the volar pad is almost fully withdrawn.

Distinctness in fingerprints makes it a good biometric trait. It has also been observed that fingerprint of identical twins are distinct [9]. Twins have been found to have different fingerprints leading to the conclusion that fingerprint formation is not completely genetically influenced.

Many applications makes use of fingerprint for identification and verification. Many such applications can be found in immigration systems, corporate public and private offices, and even in 
the entertainment industry. An example of such an application is in the United States immigration service through the Department of Homeland Security which requires that every non-immigrant seeking entry into the United States present his/her fingerprint at the point of entry and this information is stored in a secure database system known as IDENT. Corporate offices usually have server systems which contain sensitive data such as user's bank information, credit card details etc. Such servers are kept in secure locations which require authorized access. Such access is provided through biometric systems such as fingerprint or iris recognition systems. Another application can be found in some corporate offices where there is need to track the number of hours employees put into work. This takes care of the challenge of having another staff sign in on others behalf. It is also applicable in the entertainment industry, to checkmate false identity. Fingerprint has been found to be very useful as a biometric trait since it completely or partially satisfies the following characteristics:

\section{Universality:}

Virtually every human has 10 fingers and each finger comes with fingerprints found at the tip of the fingers and this makes the feature universal. In some cases there have been some users without prints which is a rare genetic condition [29]. In very few cases there exist users with more than 10 fingers with each hand having an extra digit usually located after the little finger. This condition is known as "polydactyl". This rare condition occurs in 1 out of 1000 birth [26].

\section{Distinctiveness:}

No two fingerprints are the same; twins have also been found to have distinct fingerprints features.

\section{Permanence:}

Fingerprints are permanent once the development is complete especially before birth. However, environmental factors can influence the appearance of fingerprint.

\section{Collectability:}

Fingerprint enrollment is an easy process which does not require any complex procedure however, it is an overt process and require the user to cooperative most of the time.

Performance: Fingerprint recognition come with great speed and performance in terms of matching and accuracy.

\section{Acceptability:}

Fingerprint recognition is widely accepted worldwide. 
Image A First capture

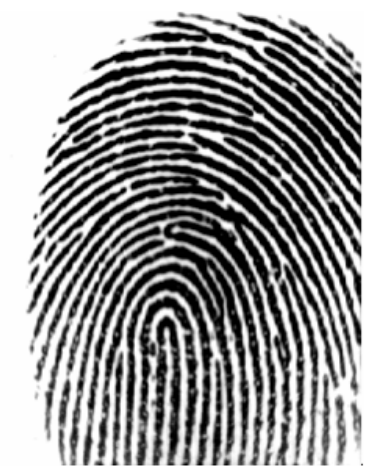

(a)

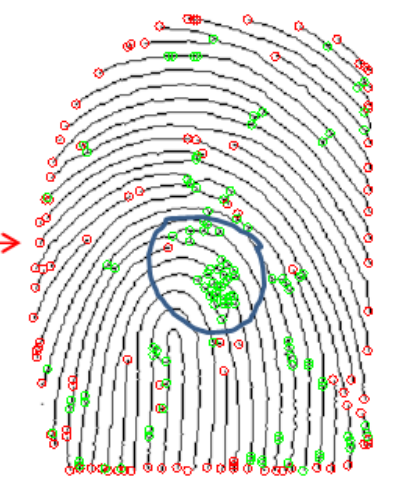

(a)

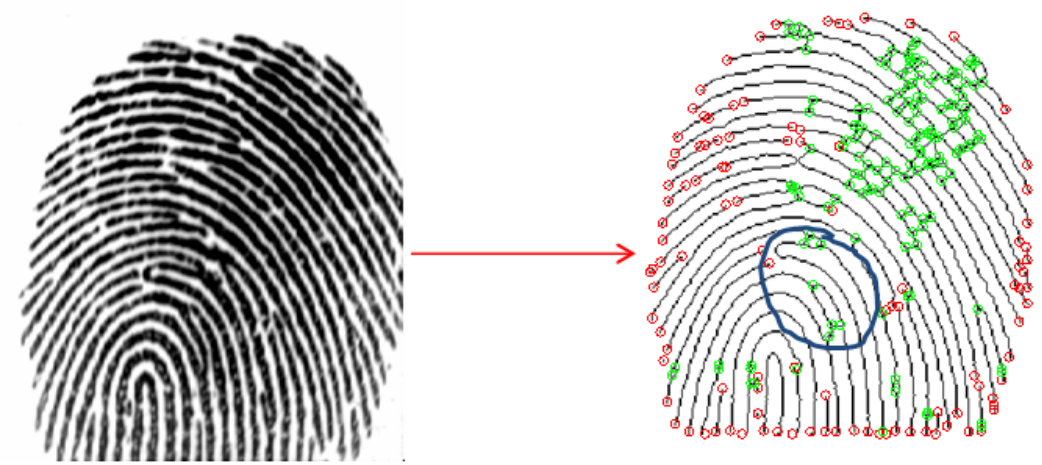

(b)

Figure 1.4: Effect of distortion on minutiae location. Red points are ridge endings while green points are mostly ridge bifurcations

\section{Circumvention:}

Users attempt to circumvent and fool fingerprint recognition system by creating synthetic fingerprint while some people have intentionally destroyed their prints. However, there are systems to checkmate synthetic fingerprint by testing for liveness of fingerprint.

While it has the many attractive characteristics it also has some disadvantages as follows.

\section{Degradation:}

Highly degraded fingerprint due to external factors or age can reduce performance drastically.

\section{Spoofing:}

Fingerprint can easily be spoofed and used by impostors. Some methods have also been used to generate fake fingerprints. However checking for features such as liveness by fingerprint systems has been used to checkmate that challenge. 


\section{Touch-based distortion:}

In touch-based fingerprint recognition, the user is required to have the finger placed on the sensor. The location of the features is not consistent after each fingerprint capture (see Figure 1.4). Touchless fingerprint recognition can be used to foil such challenges. It can be observed in Figure 1.4 that the two fingerprints which were from the same finger have varying positions of both the false and true minutiae points. In Figure 1.4(a) there were more minutiae points clustered just above the core point on the fingerprint as indicated by circular blue ring. However, there were less points around the same position in Figure 1.4(b). It can also be observed from the top right of the images that there exists more concentration of points in Figure 1.4(a) than there exist in Figure 1.4(b).

\subsection{Fingerprint characteristic}

A typical fingerprint consists of ridges and valleys while the ridges are described as friction ridges because that they provide the necessary friction for holding objects while the valleys are referred to as furrows. Fingerprints can be classified into 3 major categories: whorl, loop and arch. The classification is based on the location and number of cores and delta. The delta is the point on the fingerprint where the ridges meet while the core is the position where concentric circles formed from ridges terminate.

Fingerprints with two or more delta and core are classified as whorl. Arch fingerprint has no delta and no core while loop fingerprint contain exactly one core and one delta. Loop can also be subclassified into left loop and right loop.

\subsubsection{Fingerprint creases and importance}

Creases can be defined as irregular marks in form of stripes found on the fingerprint. They are usually formed at an angle to the ridges and valley and are typically wider than ridges. They are formed naturally during childbirth [8]. In some cases, they exist on the fingerprint due to manual labor, accident or aging. Some creases are self-inflicted by users trying to fool a fingerprint biometric system. Creases can be classified into two categories based on permanence [11].

\section{Permanent creases:}




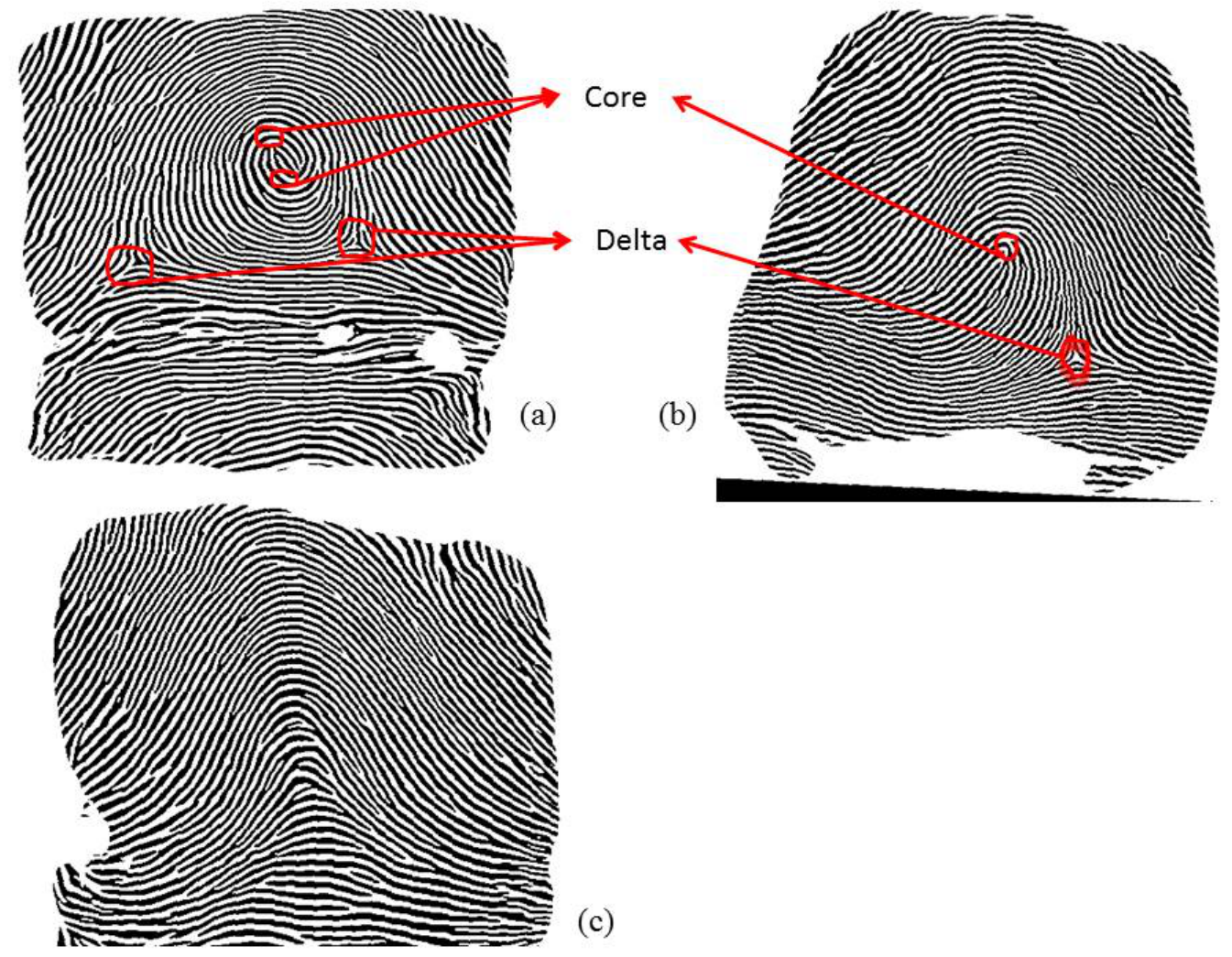

Figure 1.5: An enhanced fingerprint: (a) whorl, 2 deltas, 2 cores (b) loop, 1 delta, 1 core, (c) arch (no delta or core)

These are creases that stay on the fingerprint throughout the individual's lifetime. The most common source of permanent creases is found from birth. There is the possibility of permanent creases being genetically influenced. This is due to the fact that creases are part of the fingerprint structure which has been found to be influenced genetically. Shaham [13] describes how some fingerprint characteristics are inherited by the offspring. Some users have also tried to fool biometric systems by intentionally creating permanent crease structure by destroying the dermis layer (see Figure 1.7) or the generated layer of the epidermis of the fingerprint using sharp objects. Due to such damages the creases are not regenerated and they remain as permanent scars.

\section{Temporary creases:}

Temporary creases develop due to minimal damage to the upper portion of the epidermal layer of the fingerprint. Such a damage does not affect the regenerating cells in the dermis layer. Primary 


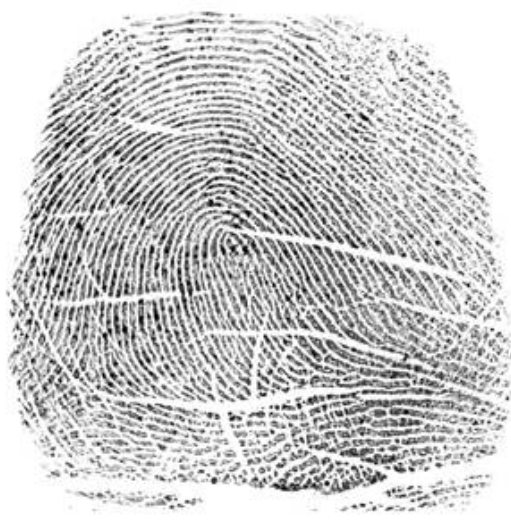

(a)

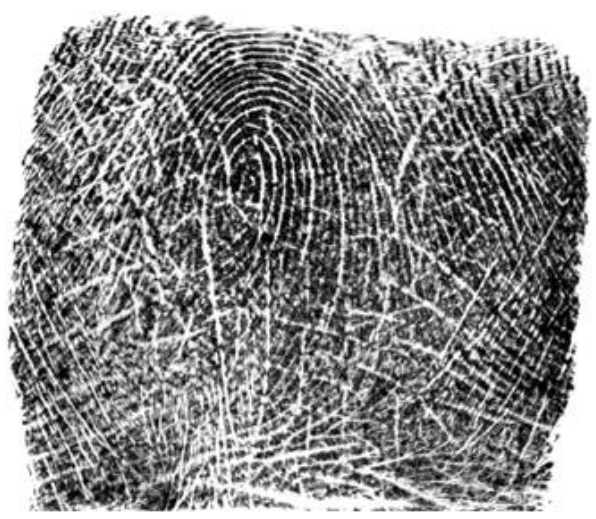

(b)

Figure 1.6: Different manifestations of creases (a) Good quality Fingerprint with obvious creases, (b) Fingerprint with less obvious creases appearing as scattered lines.

reason for such temporary creases can be traced to manual labor that involves gripping, climbing etc. Such creases usually disappear within few months of non exposure to such conditions.

Temporary creases found on fingerprints of younger people are likely to disappear within a shorter time compared to older people. Cell division is more prominent and faster in younger people while cell division is at a slower rate as aging sets in [14].

Creases are surprisingly not found on all fingers. An observation from this study suggest that most people with tendency of having creases have it on most fingers while those with no tendency for creases have creases on only one, two or at most three fingers.

Fingerprint creases could be advantageous in many circumstances. Some fingerprint images come with poor resolution and such images may not be appropriate for minutiae based matching. Creases can be obtained from poor resolution images more easily than minutiae points. Secondly, soft biometric information can be obtained from fingerprints that have creases and such information could suggest possible crease classification. Thirdly, similarity scores obtained from crease matching can be fused with scores from other fingerprint features to obtain better recognition performance. 


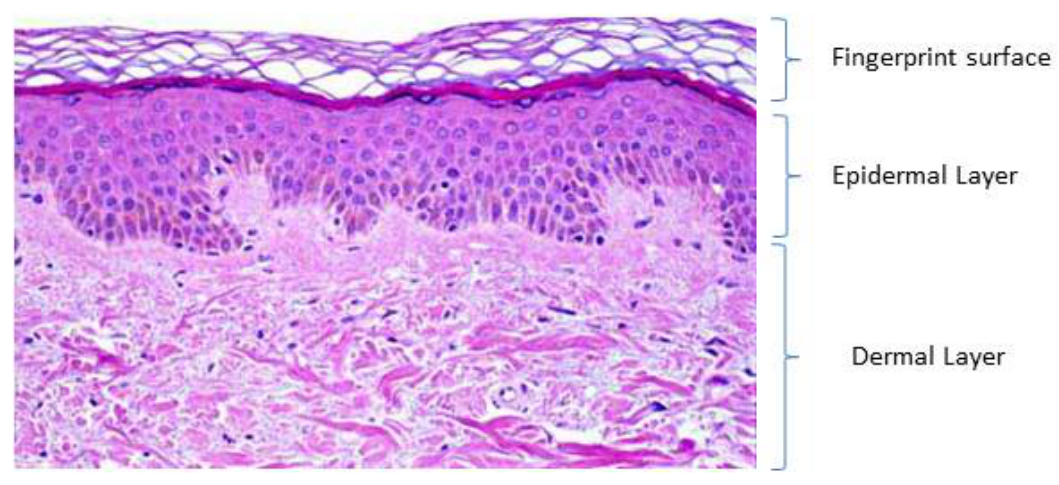

Figure 1.7: A typical fingerprint layer structure showing the fingerprint layer (dermis) and epidermis [27].

\subsection{Problem Statement and Contributions}

Most fingerprint recognition systems perform matching based on minutiae points found on the fingerprint and the performance of such systems is highly degraded by the presence of false minutiae which are incorrectly classified as real minutiae points. See Figure 1.8. It can be inferred from the images that the minutiae points in Figure 1.8(c) are actually made up of both true and false points. After the removal of the false points, the fingerprint has only the true points. The presence of false minutiae should not on its own constitute a challenge to any minutiae based system as long as there is consistency in the number of false minutiae detected from the fingerprint. However, due to the fact that there are creases that are temporary and can be short lived, this brings disparity in the number of minutiae points obtained prior and after the introduction of crease(s). A typical minutiae-based system obtains a much larger set of minutiae point with creases on the finger than when creases are not present.

An objective of this thesis is to detect the creases on the fingerprint and this is a challenging problem. Some of the challenges are described below.

Due to the complex background of a fingerprint, there is very close resemblance between the creases and the valley structure on the fingerprint, and trying to isolate the creases is usually a 




(a)

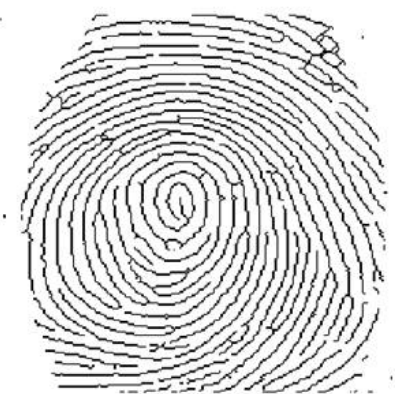

(b)

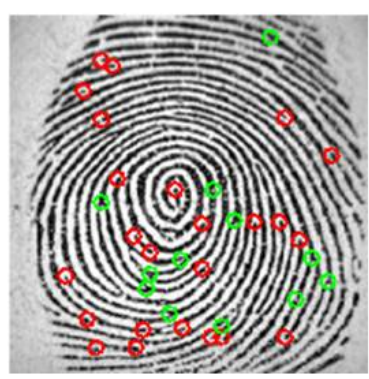

(d)

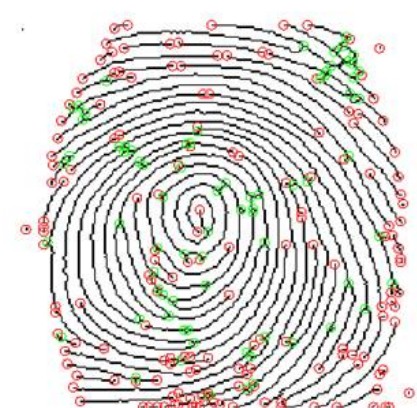

(c)
Bifurcation

Ridge ending

Figure 1.8: False minutiae and removal: (a) Original image, (b) Thinned image, (c) Thinned image with both spurious and real minutiae, (d) Original image with superimposed real/actual minutiae. 
major challenge. This study looked into two ways to approach this problem. We propose a method based on the pixel intensity of the images, we assume that creases are structures that are usually long and they appear in form of straight lines whereas valleys are oriented at an angle and not completely straight. We also have made the assumption that width of the creases is wider than that of the valleys.

We also propose a method based on the ridge orientation field of fingerprint images. We assume that the orientation field at creases becomes inconsistent with the flow of orientation field at the ridges. There is usually a spike in the angular difference at the crease while the ridge flow has a consistent angular difference along the ridge.

Very few researchers have worked on the problem of crease detection and there has not been any published papers on crease matching. This study presents results that suggest its use for recognition.

Study on creases has also been found useful to determine gender and age [10]. Experiments to confirm correlations between the creases on a person fingerprint and their age are performed in this work and results are presented that suggest possible correlations. Gender and age classification based on creases on fingerprint can be used as supporting information in forensic investigation or it can be used to narrow the search space when carrying out identification on a larger database. We anticipate other researchers to improve on this technique. 


\section{Chapter 2}

\section{Related work}

\subsection{Introduction}

Fingerprint crease detection is a challenging subject which has attracted very few researchers. We are aware of only three published papers on crease detection as of the time of this study. The nature of fingerprint texture poses a big challenge in detecting creases, and the busy background of the fingerprint makes it difficult to isolate the creases from other structures present on the fingerprint. Isolating crease(s) in a fingerprint therefore poses a problem as creases usually resemble valleys since they share similar characteristics. The valleys on fingerprints are hollow paths lying between the ridges, while the creases are known to cut through the ridges and valleys at an angle usually wide enough to differentiate it from the valleys. Visual observations suggest that most creases are at an angle of more than 20 degrees to the ridge and valley structure (see Figure 2.1).

Designing a crease detection algorithm therefore requires many assumptions to be made in order to distinctively differentiate a crease from a valley. Some of the assumptions made by other researchers include observing the basic characteristics of a crease such as the length, width, pixel intensity, etc. Zhou [10], [11], assumed a parametric representation of a crease by defining thresholds to differentiate a crease from a valley. He defined thresholds value for the pixels, crease direction, width and length in the grey scale images.

It should be noted that the performance of crease detection algorithm declines as the quality of the fingerprint drops. His algorithm performs well on good quality fingerprint with visible creases. 


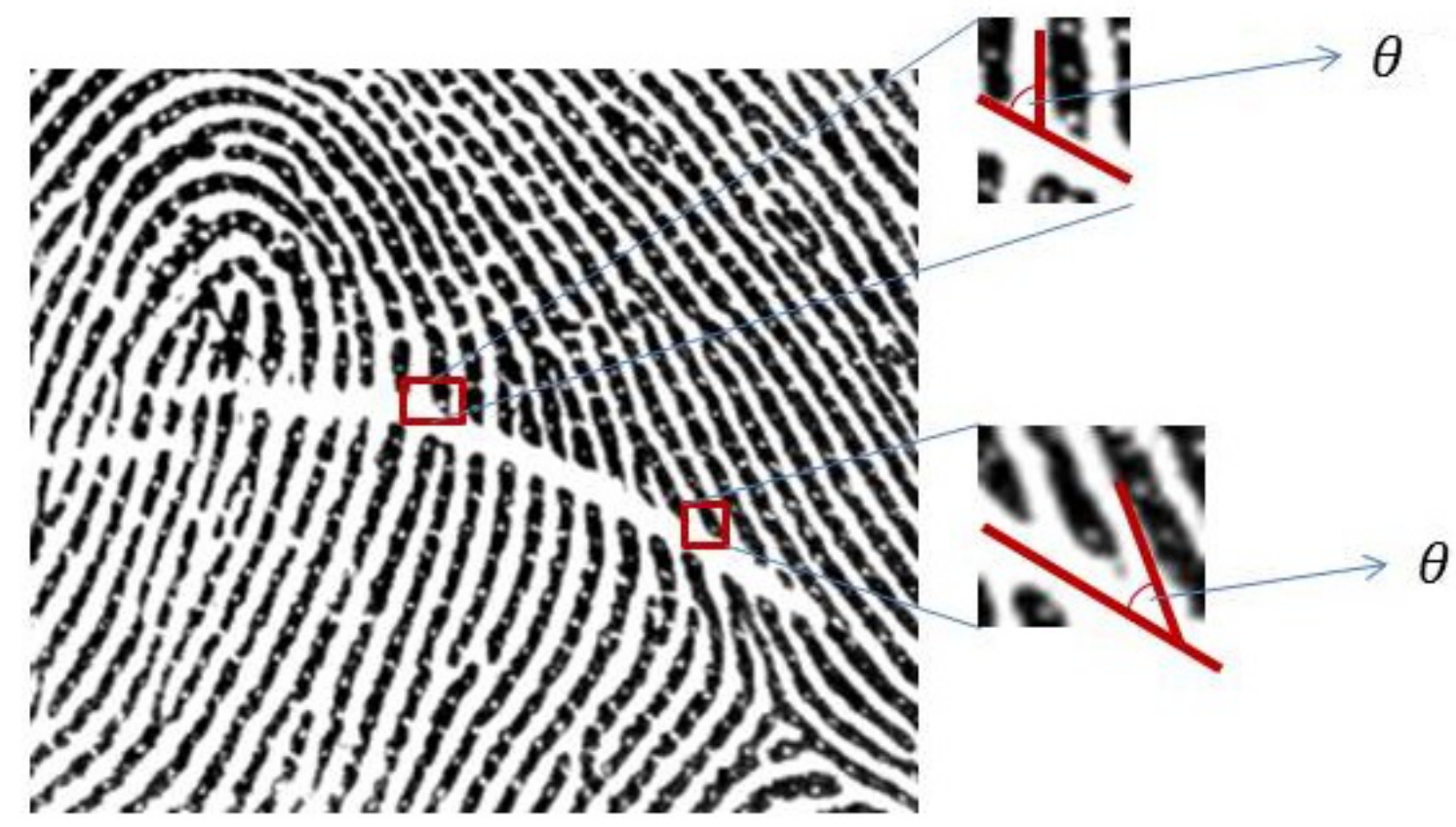

Figure 2.1: Angular difference between the fingerprint ridges and creases.

Pontus [16] described creases as straight lines on fingerprints in contrast to ridges and valleys that are not completely straight lines but are consistently bent at a certain angle. This assumption led to the application of Hough transform to detect the creases.

\subsection{Hough transform for detecting lines in objects}

Hough transform is a technique that has been widely applied in detection of lines. The concept has also been extended to circles and ellipses. It is a robust technique because its performance is not affected by image noise and gaps in feature boundary. The technique works by taking votes in a parameter space [12] in order to obtain instances of objects that fall within the same class.

The first step to obtaining lines from images is to describe a line in form of a parametric or normal notion using the equation below.

$$
p=x \cos \theta+y \sin \theta
$$

The idea is to fit set of lines to set of image points as described in Figure 2.2, where $p$ is the length of the normal from the line to the origin (see Figure 2.2). 

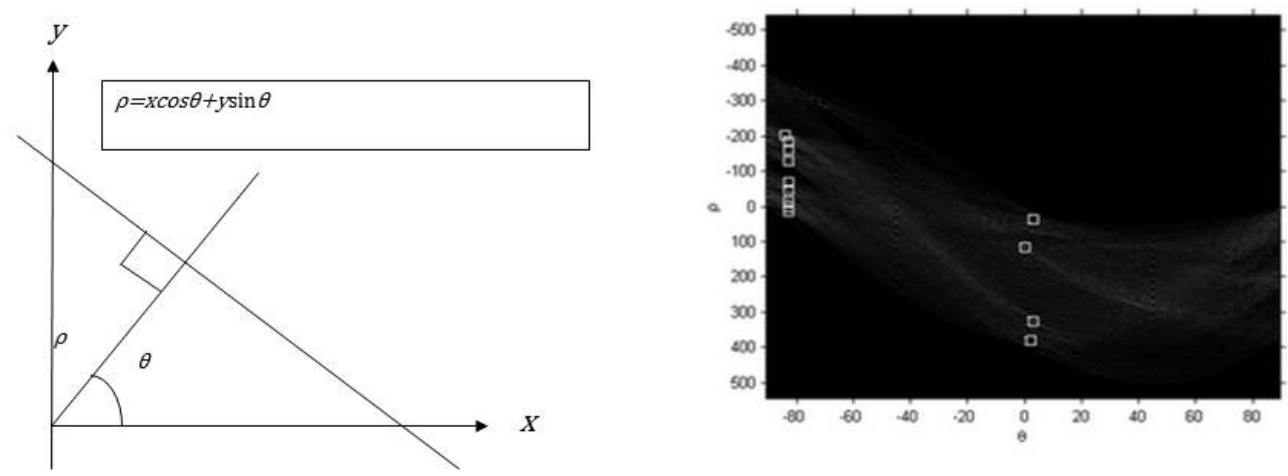

Figure 2.2: (a) Image space representation, (b) An example of Hough space representation showing the points on the space with the peak values highlighted.

The points $\left(x_{0}, y_{0}\right)$ which belong to the image space would be transformed to the sinusoidal curve that goes through the pairs $(r, \theta)$.

$$
p(\theta)=x_{0} \cos \theta+y_{0} \sin \theta
$$

If the line is vertical we would have $\theta=0$ and $x=p$. Pontus applied the concept of Hough transform to fingerprint images after performing a few preprocessing operations on the images. These include horizontal and averaging filtering (which were applied on the masked image), computing of the waterfall variance and linear symmetry, applying Gabor filter, thresholding and thinning. However some of the preprocessing steps were discarded as they did not improve the performance of the algorithm. Output of his algorithm gave the results in Figure 2.3.

We can infer from Figure 2.3 that Hough transform may not be robust enough in detecting the crease structure in an image. It can be observed that most of the creases in Figure 2.3(c) were not detected even if preprocessing was done before applying the Hough transform. The only crease detected is highlighted in red. Zhou [11] supported the conclusion that due to the similarity between the creases and the valleys it may be impossible to obtain creases using the Hough transform. 



Figure 2.3: Application of Hough transform on fingerprint images[15] (a) Original image, (b) Hough transform Space (c) Line detected after applying Hough transform.

\subsection{Crease representation as parameterized rectangle}

The structure of creases can be completely described as parameterized rectangles according to Zhou [10], [11]. A parameterized rectangle of a crease is defined in Figure 2.4.

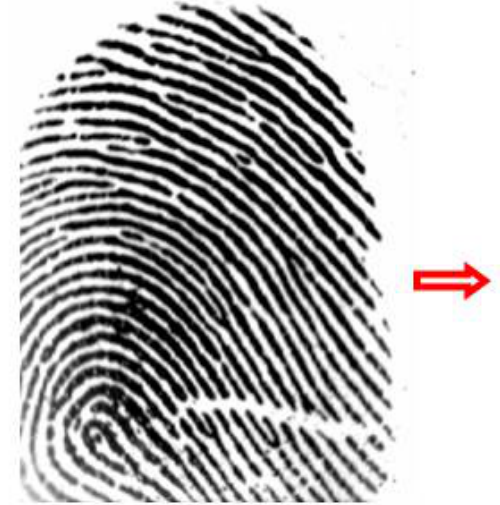

(a)

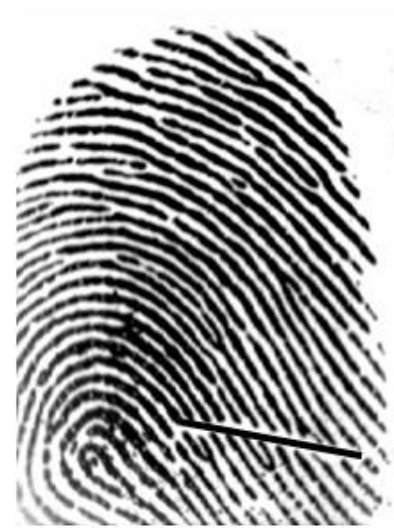

(b)

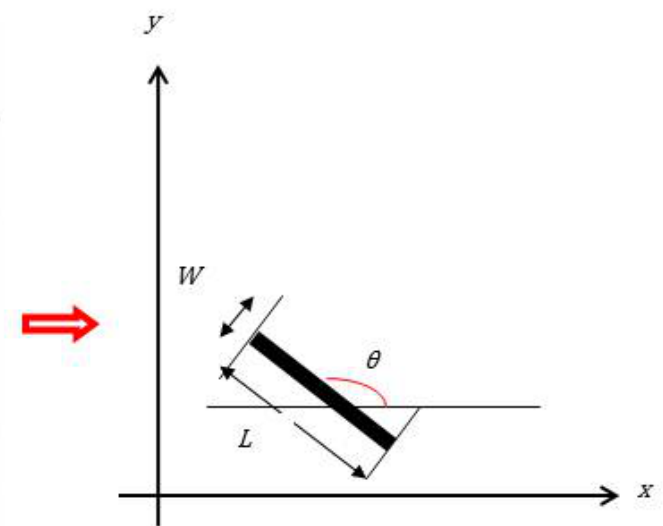

(c)

Figure 2.4: (a) A fingerprint with a crease structure. (b) A crease represented by a rectangle (c) A parameterized triangle in the $x-y$ domain.

Creases usually have constant width but varying length and direction. Zhou tried to isolate a crease from a valley by defining thresholds on the creases (see Figure 2.4(c)). He made some assumptions based on the the parameters of a rectangle which include the length, width, grey scale 
value and pixel intensity.

$$
\sum_{x, y \in L} \nabla I(x, y)<t_{1}
$$

Equation (2.3) suggests that the change in gray value within creases should be less than $t_{1}$

$$
\sum_{x, y \in L} \nabla|\alpha(x, y)-\theta|>t_{2}
$$

Equation (2.4) suggests that the crease direction with respect to ridge direction is greater than $t_{2}$

$$
t_{3}<w<t_{4}
$$

Equation (2.5) suggests that the width of a crease is bounded by $t_{3}$ and $t_{4}$

$$
l>t_{5}
$$

Equation (2.6) suggests that the length of a crease is greater than $t_{5}$

$$
m I(x, y)>t_{6}
$$

Equation (2.7) suggests that the average grey value of a crease is greater than $t_{6}$

By applying the above constraints on rectangles extracted from images containing creases, it is likely that most of the creases will be classified appropriately. In order to obtain the creases based on the above equations, he designed an optimal filter as below.

For this particular problem, Zhou designed an optimal filter based on the Gaussian function. The function is useful when applied to the crease and its adjacent valley structure. The optimal filter is therefore the filter with the maximum response. In this particular problem the width and direction of the crease are considered to obtain the filter with the greatest response. It is also assumed that the valley and ridge have the same width represented as $w_{0}$. The steps involved in applying the Gaussian filter are as follows:

- Denote a crease using a Gaussian function described as $G_{x}\left(0, \sigma_{1}^{2}\right)$, and denote the valley using a Gaussian function described as $G_{x}\left(0, \sigma_{2}^{2}\right)$. Since the goal is to obtain the best filter, it is necessary to maximize $G_{x}\left(0, \sigma_{1}^{2}\right)$ while $G_{x}\left(0, \sigma_{2}^{2}\right)$ is minimized.

It follows that the optimal filter can be described as

$$
s(x)=G_{x}\left(0, \sigma_{1}^{2}\right)-G_{x}\left(0, \sigma_{2}^{2}\right)
$$




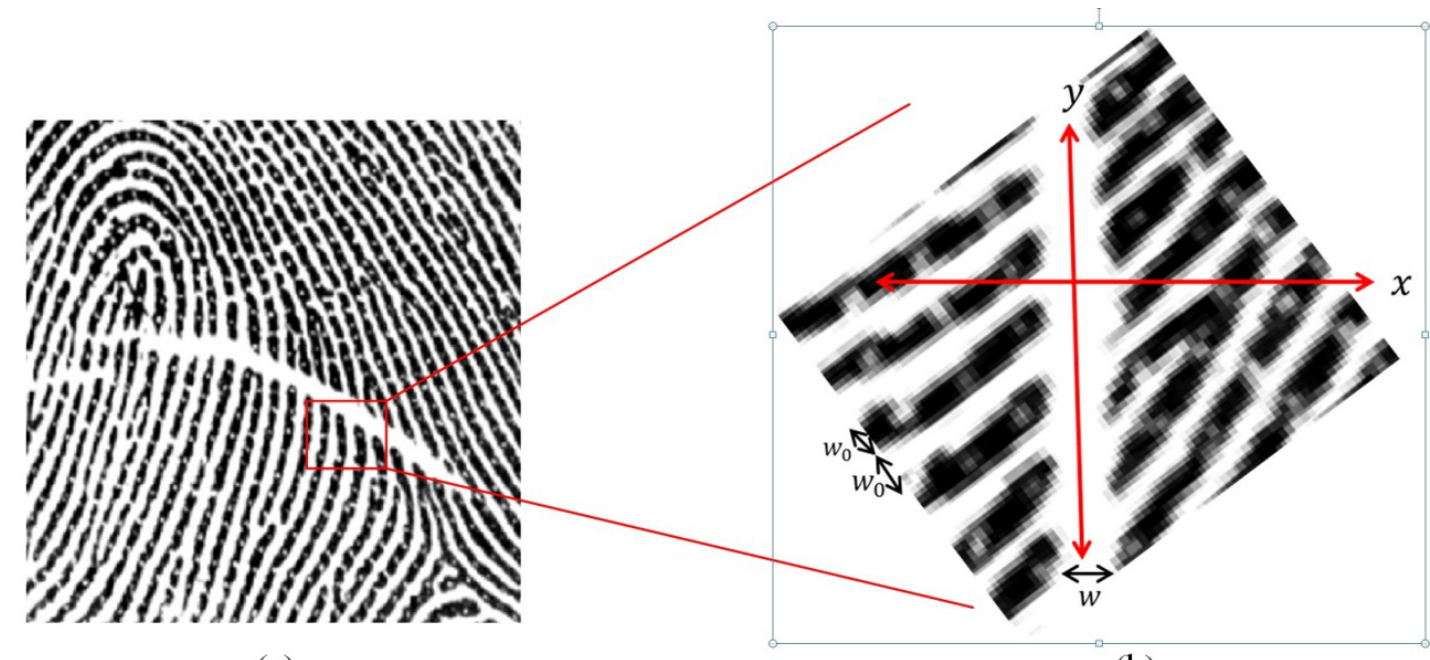

(a)

(b)

Figure 2.5: (a) Fingerprint creases (b) Creases showing the detailed width representation of ridge and valley as being the same.

- He approximated the above filter by assuming a two-order derivative Gaussian function given approximate variance which is described below.

$$
F(x, y)=\text { const.exp }\left\{-\frac{x^{2}+\eta y^{2}}{2 T^{2}}\right\}\left(T^{2}-x^{2}\right)
$$

In the above $T$ is the Threshold while $\eta$ is an aspect-ratio parameter.

- He rotated the above function through different directions as shown below:

$$
F_{\gamma}(x, y)=\text { const.exp }\left\{-\frac{u^{2}+\eta v^{2}}{2 T^{2}}\right\}\left(T^{2}-u^{2}\right)
$$

Here, $u=x \cos \gamma+y \sin \gamma$ and $v=-x \sin \gamma+y \cos \gamma$.

Applying the above while varying the threshold results in a maximum response for both creases and ridges. The maximum value obtained for crease would be much greater than that for valleys. This provides us with information about the threshold with the most discriminative tendency for valleys and the creases.

Invoking equation (2.10) for 12 channels produced 12 filters. For each channel, thresholding was performed and binary responses were obtained. Channel responses with gray level greater than the specified threshold are assumed to belong to crease. PCA was applied to each channel to 
estimate the parameters which include the width, length, and direction $\theta$ of the crease. Combining each crease in the different channels produced the results in Figure 2.6.
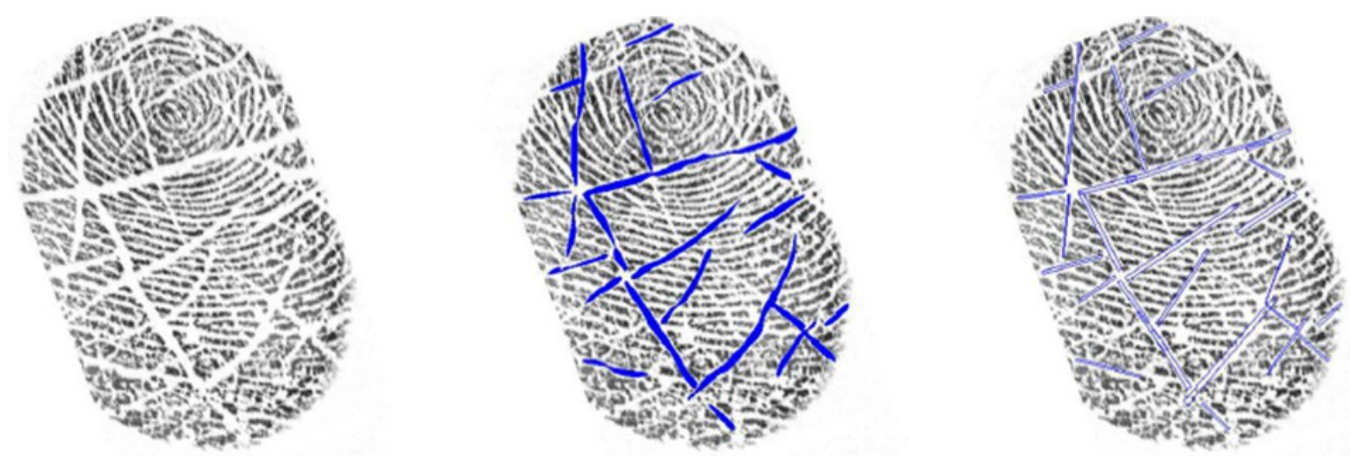

Figure 2.6: Output of crease detection algorithm obtained from [11] 


\section{Chapter 3}

\section{Matching and Classification}

\subsection{Fingerprint matching based on creases}

\subsubsection{Introduction}

Fingerprint matching has been widely studied especially matching methods based on minutiae. However, matching based on creases has not been studied. Matching involves comparing two samples and generating a match score which can be a similarity score or a distance score. A large similarity score indicates that the two samples are very similar while a small similarity score indicates dissimilarity of the two samples. A low distance score indicates that the two samples are very similar while a large distance score indicate dissimilarity.

Matching fingerprints is not a trivial problem. Factors such as rotation, displacement, nonlinear distortion and feature extraction errors [5] contribute to poor performance of fingerprint recognition systems by increasing the intra-class variations.

Fingerprint matching can be correlation-based, minutiae-based, or non-minutiae feature-based matching. Correlation-based method involves the superimposition of two fingerprints images and generating matching score based on the corresponding pixels on the respective fingerprints. Minutiae-based method involves comparing minutiae points extracted from two fingerprints. Nonminutiae based methods involve matching based on features such as the ridges found on the fingerprint. However such features may not be stable enough to provide good performance.

Bazen [28] performed correlation-based matching using gray-level information extracted from 
the fingerprints. It is assumed that gray-level information is more discriminative than minutiae points in some situations. Minutiae points may be difficult to extract especially from poor fingerprints while the gray-level information extraction may not be as adversely affected by quality of fingerprints. The system performed with an equal error rate (EER) of $7.98 \%$ on a database of 880 fingerprints. Correlation-based methods are very resource intensive due to the fact that a large number of pixel positions on the image are considered. Minutiae-based matching typically results in high matching performance.

\subsubsection{Method}

In order to assess the matching performance due to creases, the following procedure was adopted. First, a set of 101 fingerprints were considered. Each fingerprint had two samples each. One sample was assigned to the template database and the other was assigned to the query database. The two samples of each fingerprints were acquired at different time instances. Samples are all gray-scaled images of size 800 x 748 pixels. Matching based on creases in this work is an exploratory research to determine if creases can be used for recognition. The matching algorithm to compare two samples, one from the template database and the other from the query database, consists of the following steps:

- Creases on the two fingerprint samples in the query and template databases were manually marked see Figure 3.1.

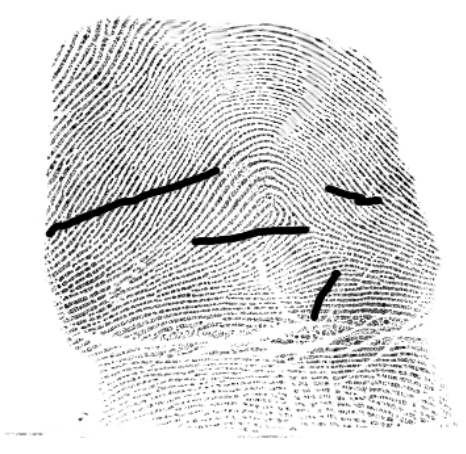

Figure 3.1: An example of manually marked crease

- Fingerprint orientation fields were extracted from the two samples, $Q$ and $T$. 
- Each crease was denoted as a set of points sampled at 10 pixel intervals. Let the query, $Q$, and the template, $T$, have a total of $n$ and $m$ sampled crease points, respectively. Each point has 3 values: location coordinates denoted by $x$ and $y$, and orientation, $t$. The orientation, $t$, is based on the extracted orientation field.

- Given $Q=\left\{Q_{1}, Q_{2}, Q_{3}, \ldots, Q_{n}\right\}$

and $T=\left\{T_{1}, T_{2}, T_{3}, \ldots, T_{m}\right\}$

where $n$ and $m$ are the total number of points in $Q$ and $T$ respectively.

$Q_{i}=\left\{x_{Q}^{i}, y_{Q}^{i}, t_{Q}^{i}\right\}$,

and $T_{j}=\left\{x_{T}^{j}, y_{T}^{j}, t_{T}^{j}\right\}$.

For $i=1$ to $n$ and $j=1$ to $m$

We obtain the transformation parameters between $Q_{i}$ and $T_{j}$ as follows:

$t_{x}=x_{T}^{j}-x_{Q}^{i}$

$t_{y}=y_{T}^{j}-y_{Q}^{i}$

$t_{t}=\operatorname{ang}\left[t_{T}^{j}-t_{Q}^{i}\right]$

$A_{i j}=\left\{t_{x}, t_{y}, t_{t}\right\}$

$A_{i j}$ is applied to all points in $Q$ as per the following equation in order to transform the points using the estimated transformation parameters.

For $k=1$ to $n$

$x_{k}^{Q}=\left\{x_{k}^{Q}-x_{i}^{Q}\right\}$ Cost $_{t}+\left\{y_{k}^{Q}-y_{i}^{Q}\right\}$ Sint $_{t}+t_{x}+x_{i}^{Q}$

$y_{k}^{Q}=-\left\{x_{k}^{Q}-x_{i}^{Q}\right\}$ Sint $_{t}+\left\{y_{k}^{Q}-y_{i}^{Q}\right\}$ Cost $_{t}+t_{y}+y_{i}^{Q}$

end $k$

We now find the corresponding points between $Q$ and $T$

if $\left(N_{i j}>n_{\max }\right)$

$N_{\max }=n_{i j}$

$S_{i, j}=A_{i j}$

end $(j)$

end $(i)$

- The highest value for $S_{i, j}$ (bestcount) is recorded and similarity score is obtained based on 
the following equation:

$$
\text { Score }=\left(\text { bestcount }^{2} * 100\right) /(n * m)
$$

\subsubsection{Results}

False accept rate (FAR) and false reject rates (FRR) were computed. Receiver operating characteristic (ROC) curve was generated from the FAR and FRR to describe the performance of the system as can be seen in Figure 3.2.

Distribution graphs of the scores of genuine and impostor pairs were also generated in Figure 3.3.

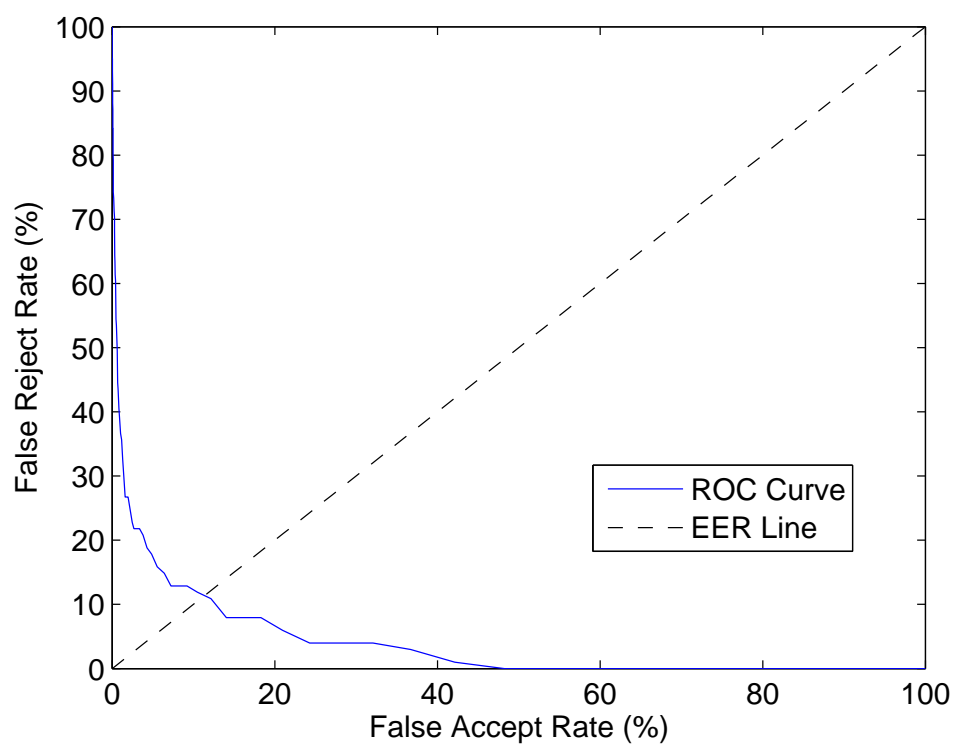

Figure 3.2: ROC curve for FAR and FRR

Matching of fingerprints in our experiments was based on points located on the creases. It was discovered that utilizing all the points covered by creases would be computationally impossible. Points generated from each fingerprint image were stored in text files for matching using the point to point matching algorithm described above. Some of the files had up to 1,500 points for each fingerprint and matching two files with such a large number of points would be computationally demanding. To reduce the computation required, the creases were sampled at 10-pixel intervals. Results in Figures 3.3 and 3.2 indicate the performance at a sampling interval of 10 pixels. An EER of $15 \%$ was obtained. 


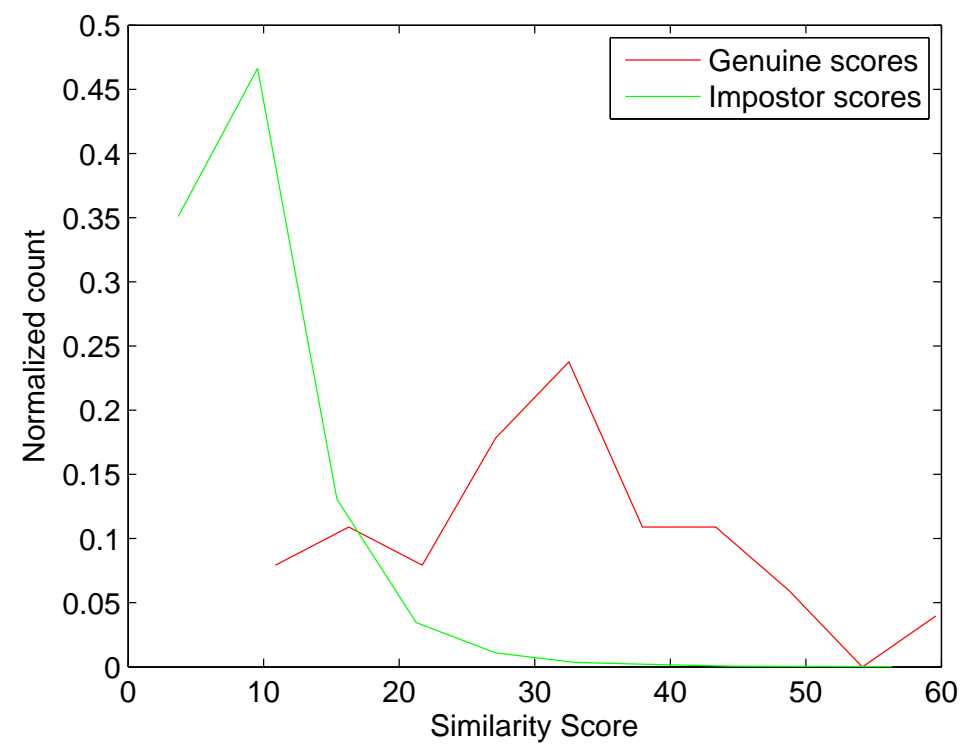

Figure 3.3: Distribution of normalized genuine and impostor scores for the crease matching algorithm

Experiments were also performed by sampling the creases at 5-pixel intervals. This was to confirm if there could be an improvement in performance. This computation of scores based on 5-pixel intervals was computationally very demanding. Results presented indicated a lower(better) equal error rate (EER) of about $8 \%$. Receiver operating characteristic (ROC) curve and distribution graphs of the genuine and impostor scores are presented in Figures 3.4 and 3.5, respectively.

Experiments were also conducted based on minutiae points. Database contained same number of images and files as used in the previous experiment using crease. VeriFinger, a robust fingerprint recognition software by Neurotechnology, was employed to compute the performance based on minutiae points. Results indicates an equal error rate of less than $1 \%$.

Experiments were also conducted to confirm if there exists a relationship between the number of creases found on the fingerprint and the matching performance of the system. The same database used for matching was explored for this experiment. Firstly, the number of creases found on fingerprints were recorded and the histogram is presented in Figure 3.6. From the histogram it can be inferred that the mean number of creases is about 3 . The number of fingerprint with creases less that or equal to 3 and those greater than 3 is 103 and 99, respectively. Two databases were created: fingerprints with 3 creases and less, and those with more than 3 creases formed the two databases. 




Figure 3.4: ROC curve for FAR and FRR



Figure 3.5: Distribution of normalized genuine and impostor scores for the crease matching algorithm

Each database contains gallery and query templates. Match scores, false acceptance rates, and false rejection rates were computed in the process. Receiver operating characteristic (ROC) curves to measure performance for both databases were also generated and are presented in Figures 3.7 and 3.8. The ROC curve indicates an EER performance of about $12 \%$ for each of the databases. 




Figure 3.6: Distribution of number of creases found on fingerprint

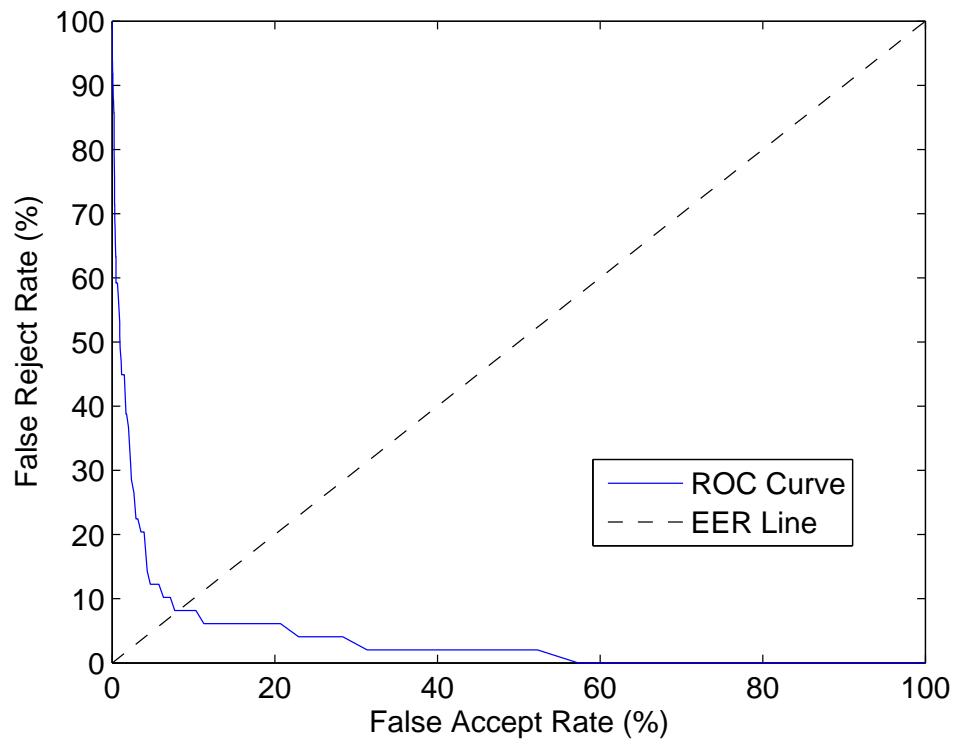

Figure 3.7: ROC Curve based on creases less than or equal 3

It can be concluded that there may not be a relationship between the performance of the systems based on the number of creases found on them. 


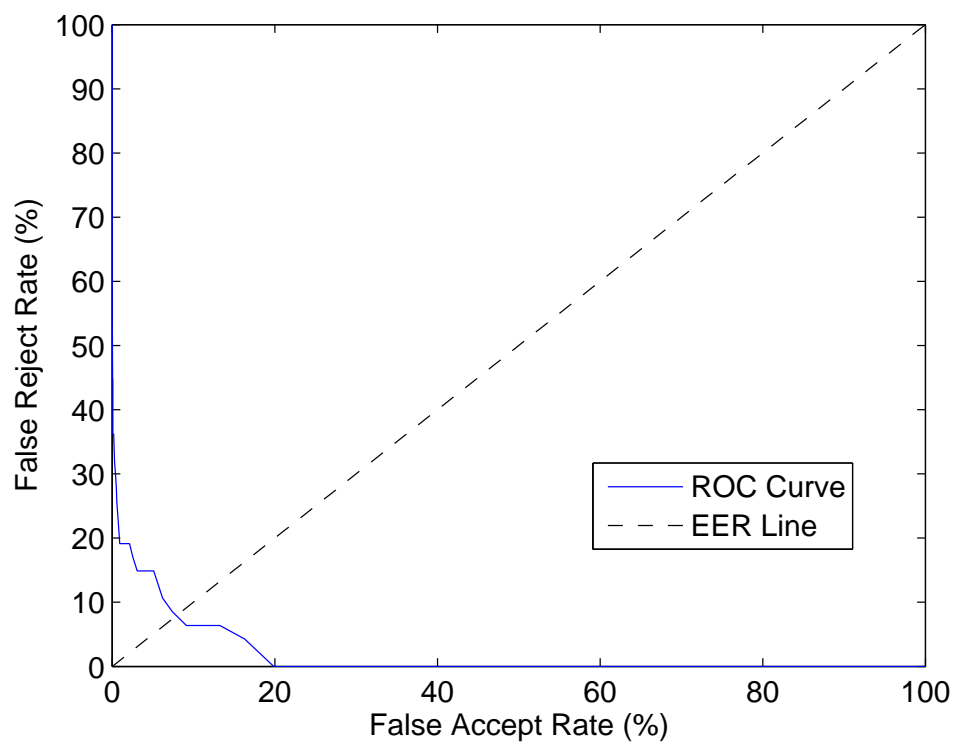

Figure 3.8: ROC Curve based on creases greater than 3 


\subsection{Classification of age groups based on creases}

\subsubsection{Introduction}

Classification of a population based on gender and age has been widely used in socio-political analysis, classification can however also be based on different biometric traits including ridge and valley thickness, ridge count, pattern type, white lines etc. in the case of fingerprints.

An important benefit of gender classification is to reduce the search space in a biometric database that has a large number of identities. An example can be a government database with millions of fingerprint templates. Searching through such a database would require a scheme to narrow the search space. Having prior knowledge of the gender or age category of the sample based on the crease pattern would be very beneficial in reducing computation and computer resources during recognition.

Badawi [24] performed experiments on gender classification based on a combination of fingerprint traits including fingerprint ridge count, ridge and valley thickness ratio, and pattern type concordance. The results obtained indicated a $80.39 \%$ accuracy using fuzzy c-means, $86.5 \%$ accuracy using linear discriminant analysis and $88.5 \%$ using neural network on a database of 22,000 images.

In our work, we analyzed the correlation between creases and age. As of the time of performing this work, there is no known research on the correlation of fingerprint with different age groups and this serves as the first attempt.

A demerit of this analysis is that fingerprint creases are not universal, and hence this study is applicable to only groups of people with creases.

\subsubsection{Method}

As described earlier, the feature for our classification is based on creases which are the irregular striped lines/scars found in some fingerprints. Pixel counts and geometric area occupied by creases were considered. Unlike ridge count which is pretty easy to define, creases may however be difficult to identify and label in some cases. Some creases appear to be very thin with a small 
width while some other creases are scattered all over the fingertip. Portions of the fingerprint with creases were manually marked, and the area occupied by the creases was computed by calculating the number of pixels occupied by the creases. It was also observed that the area occupied by a fingerprint in each image varies across the database. Some fingerprints occupied up to $30 \%$ of the total image area while some occupied up to $70 \%$ of the total image area. Using a direct count of the pixels occupied may not be a true reflection of the density of creases in a fingerprint. Therefore, the pixel count was normalized by the size of the fingerprint. This was a better measure of the area covered by the creases irrespective of the size of the image.
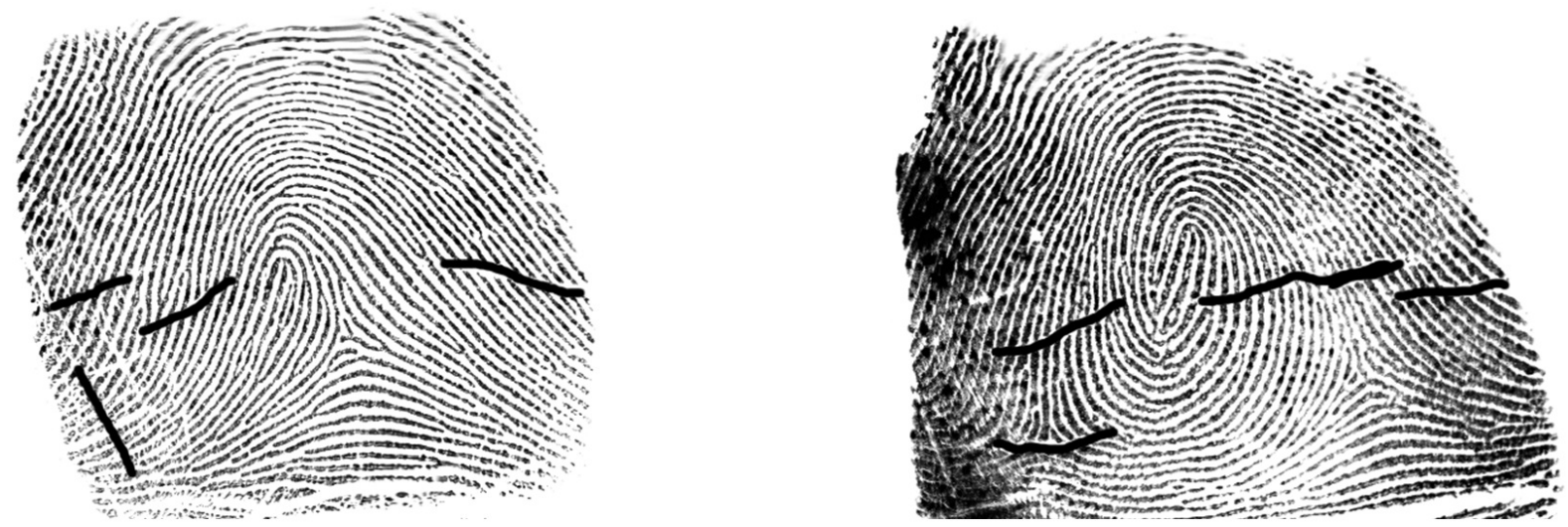

Figure 3.9: Images showing the regions covered by creases manually marked out.

The following steps were undertaken:

- We replaced all 0-value pixels with a value of 1 .

- We manually marked the creases. Manually marked creases are assigned a pixel value of 0 . This allows us to easily compute the number of crease pixels.

- We compute the region of interest for each image and then compute the total pixels constituting the region of interest. 



Figure 3.10: Histogram showing left thumb and left index fingers (ages 0-20, 50-80)
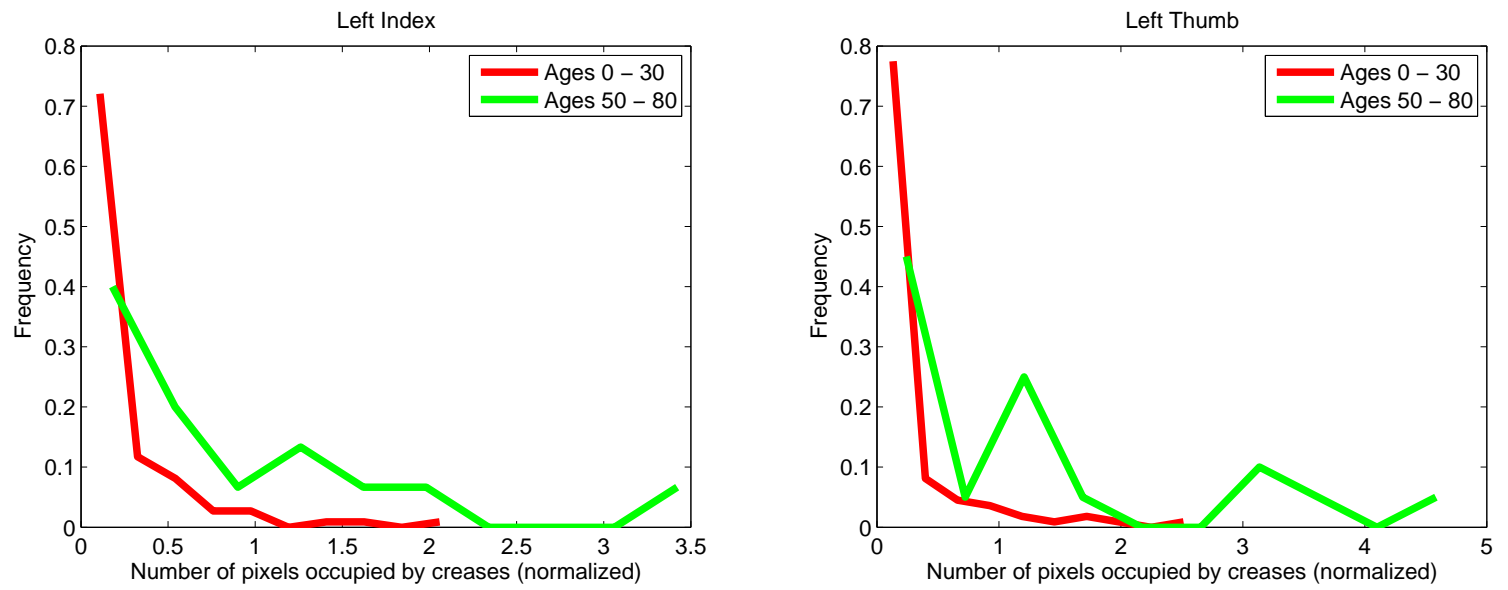

Figure 3.11: Histogram showing left thumb and left index finger (ages 0-30, 50-80)

- We normalize the pixel count in step 2 with the pixel count in step 3 and multiply the result by 100

For this problem, two different databases were created: left thumb images and left index images. The major reason was to confirm consistency in the results in both fingers. 

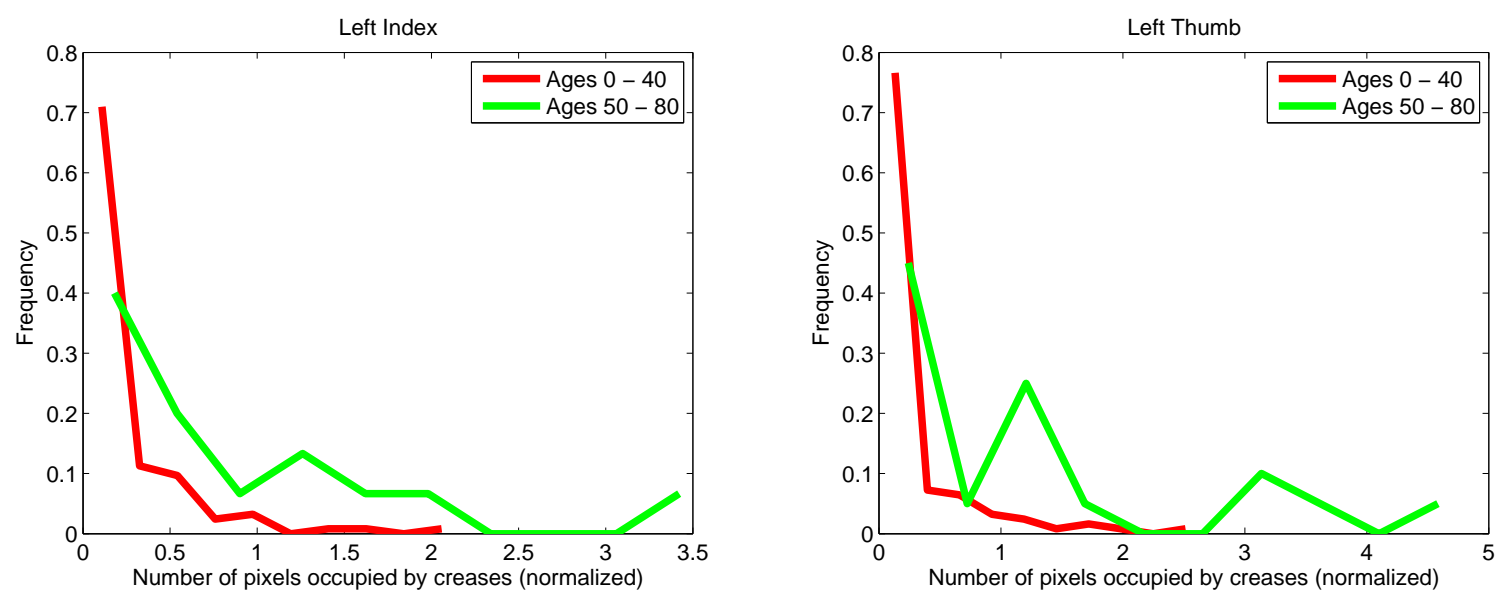

Figure 3.12: Histogram showing left thumb and left index finger (ages 0-40, 50-80)

\subsubsection{Results}

The left thumb database is made up of 152 samples while the left index is made up of 146 samples. The plots were based on the above data for age and the graphs in Figures 3.10, 3.11, and 3.12, were generated. From the figures, it can be inferred that potential correlations exist between age and the extent of creases found on the fingerprint. The result is also consistent in the two different databases used for the experiment. We can infer that more people in the age group [040] have fewer creases than people in the age group [41-85]. This may be due to impact of age or manual labor. Creases developed on fingers of old people are less likely to disappear as cell division and growth is highly reduced.

Analyzing the plots of the left index finger in Figure 3.12, it can be inferred that the normalized pixels corresponding to the creases for those in ages 0 - 40 had a peak value of 2.1 while those in ages 50 - 80 has a peak value of 3.4. Analyzing the left thumb in Figure 3.12, a larger area is occupied by the creases in general with the normalized size up to 4.5 for ages $40-80$ and a peak value of 2.5 for ages $0-40$.

It can be hypothesized that left index and thumb fingerprint with normalized crease area greater than 2.1 and 2.5, respectively, would likely be classified as a person belonging to the age category of $50-80$. We can also observe that the plots in Figure 3.11 (Left Index) and Figure 3.11 (Left Thumb) which considered the age category 0-30 and 50-80, are very similar to Figure 3.12 (Left Index) and Figure 3.12 (Left Thumb). However, there is a big contrast between Figure 3.10 (Left 
Index) and Figure 3.10 (Left Thumb) which consists of age group 0- 20 and 50-80. It can be observed from Figure 3.10 (Left Index) and Figure 3.10 (Left Thumb) that the area occupied by the creases for the age group 0 - 20 years peaks at 0.7 and 1.7 , respectively. We can conclude that most subjects between the ages 0 and 20 have fewer crease pixels. The above suggests that a possible correlation exists between age categories and the number of crease pixels present on fingerprints.

Furthermore, we used statistical methods in order to confirm the reliability of the differences in results obtained between the age groups. The primary reason is to eliminate any doubt suggesting that the variation in the mean of different age groups occurred by chance. Results further confirm that mean value of area covered by creases are potential indicators for variation in crease patterns between different age groups. Data analysis was performed using one-way ANOVA which is a robust statistical method used for analyzing data with more than two groups.

Each of Figures 3.13, 3.14, 3.15, 3.16, describes the sum, average and variance for each group. Test results of one-way ANOVA are presented on the lower portion of the figures. The result includes values for the sum of squares, degree of freedom, mean of squares, calculated $F$-values, $P$-value and the $F$ crit-value.

It can be inferred from Figure 3.13, that the $F$-values is much greater than the $F$ crit-value. Furthermore it can be observed that the p-value is much lower than $\alpha=0.5$ which suggests that something significant also occurs between the groups. Hence, we have to reject the null hypothesis and conclude that the three groups have unequal means.

Similar result can also be observed in Figure 3.14. The value for $F-$ crit is 2.637 which is much lower than the $F$-value of 19.46 . The $P$-value is also much lower than $\alpha=0.5$ and hence we can reject the null hypothesis.

The experiments were carried out on two groups: first group considered overlapping age ranges (see Figures 3.13, 3.14) while the second group considered distinct age groups (see Figures 3.15, 3.16). Both groups had similar results thereby suggesting that we have to reject the null hypothesis and conclude that the variation in the mean values were not by chance but confirms that there is a possible relationship between age groups and extent of creases on fingerprints. 


\begin{tabular}{|c|c|c|c|c|c|c|}
\hline \multicolumn{7}{|l|}{ SUMMARY } \\
\hline Groups & Count & Sum & Average & Variance & & \\
\hline $0-20$ years & 28 & 4.12068355 & 0.14717 & 0.05118 & & \\
\hline $0-30$ years & 110 & 21.3566154 & 0.19415 & 0.1252 & & \\
\hline $0-40$ years & 124 & 25.0172492 & 0.20175 & 0.12202 & & \\
\hline $50-80$ years & 15 & 12.5701307 & 0.83801 & 1.00943 & & \\
\hline \multicolumn{7}{|l|}{ ANOVA } \\
\hline Source of Variation & SS & $d f$ & $M S$ & $F$ & $P$-value & F crit \\
\hline Between Groups & 5.97604 & 3 & 1.99201 & 12.3122 & $1.4 \mathrm{E}-07$ & 2.63767 \\
\hline Within Groups & 44.1692 & 273 & 0.16179 & & & \\
\hline Total & 50.1452 & 276 & & & & \\
\hline
\end{tabular}

Figure 3.13: ANOVA analysis of left index fingers (overlapping age groups)

\begin{tabular}{|c|c|c|c|c|c|c|}
\hline \multicolumn{7}{|l|}{ SUMMARY } \\
\hline Groups & Count & Sum & Average & Variance & & \\
\hline $0-20$ years & 28 & 4.737403 & 0.169193 & 0.136055 & & \\
\hline $0-30$ years & 110 & 24.89493 & 0.226318 & 0.22863 & & \\
\hline $0-40$ years & 124 & 28.47434 & 0.229632 & 0.219602 & & \\
\hline $50-80$ years & 20 & 24.84222 & 1.242111 & 1.941711 & & \\
\hline \multicolumn{7}{|l|}{ ANOVA } \\
\hline Source of Variation & SS & $d f$ & $M S$ & $F$ & P-value & F crit \\
\hline Between Groups & 19.4321 & 3 & 6.477367 & 19.46759 & $1.74 \mathrm{E}-11$ & 2.637076 \\
\hline Within Groups & 92.49773 & 278 & 0.332726 & & & \\
\hline Total & 111.9298 & 281 & & & & \\
\hline
\end{tabular}

Figure 3.14: ANOVA analysis of left thumb fingers (overlapping age groups)

\begin{tabular}{|c|c|c|c|c|c|c|}
\hline \multicolumn{7}{|l|}{ SUMMARY } \\
\hline Groups & Count & Sum & Average & Variance & & \\
\hline $0-20$ years & 49 & 11.82535 & 0.241334 & 0.15234 & & \\
\hline $21-50$ years & 83 & 18.33771 & 0.220936 & 0.18506 & & \\
\hline $51-80$ years & 14 & 12.56972 & 0.897837 & 1.029257 & & \\
\hline \multicolumn{7}{|l|}{ ANOVA } \\
\hline Source of Variation & SS & $d f$ & MS & $F$ & P-value & F crit \\
\hline Between Groups & 5.683418 & 2 & 2.841709 & 11.32958 & $2.71 \mathrm{E}-05$ & 3.059376 \\
\hline Within Groups & 35.86755 & 143 & 0.250822 & & & \\
\hline Total & 41.55097 & 145 & & & & \\
\hline
\end{tabular}

Figure 3.15: ANOVA analysis of left index fingers (distinct age groups) 


\begin{tabular}{|c|c|c|c|c|c|c|}
\hline \multicolumn{7}{|l|}{ SUMMARY } \\
\hline Groups & Count & Sum & Average & Variance & & \\
\hline $0-20$ years & 49 & 11.3124 & 0.23086 & 0.23592 & & \\
\hline $21-50$ years & 84 & 25.099 & 0.2988 & 0.34405 & & \\
\hline $51-80$ years & 19 & 24.8417 & 1.30746 & 1.95943 & & \\
\hline \multicolumn{7}{|l|}{ ANOVA } \\
\hline Source of Variation & SS & $d f$ & MS & $F$ & P-value & F crit \\
\hline Between Groups & 17.9068 & 2 & 8.95342 & 17.752 & $1.2 \mathrm{E}-07$ & 3.05678 \\
\hline Within Groups & 75.1499 & 149 & 0.50436 & & & \\
\hline Total & 93.0567 & 151 & & & & \\
\hline
\end{tabular}

Figure 3.16: ANOVA analysis of left thumb fingers (distinct age groups) 


\section{Chapter 4}

\section{Automated fingerprint crease detection techniques}

The automated fingerprint crease detection techniques proposed in this study rely on some concepts from the field of pattern recognition. Two methods are proposed in this work. The first method is based on the pixel intensity of the creases, and the second is based on the pattern of fingerprint orientation fields.

The experiments were conducted on a fingerprint database containing 1,487 subjects, with each subject providing 2 samples each. This yielded a database of 31,350 images. The number of images with noticeable creases is 1,068 , which is about $3 \%$ of the total number of images. This small percentage indicates that creases are not a commonly observed feature in fingerprints.

\subsection{Pixel intensity based method}




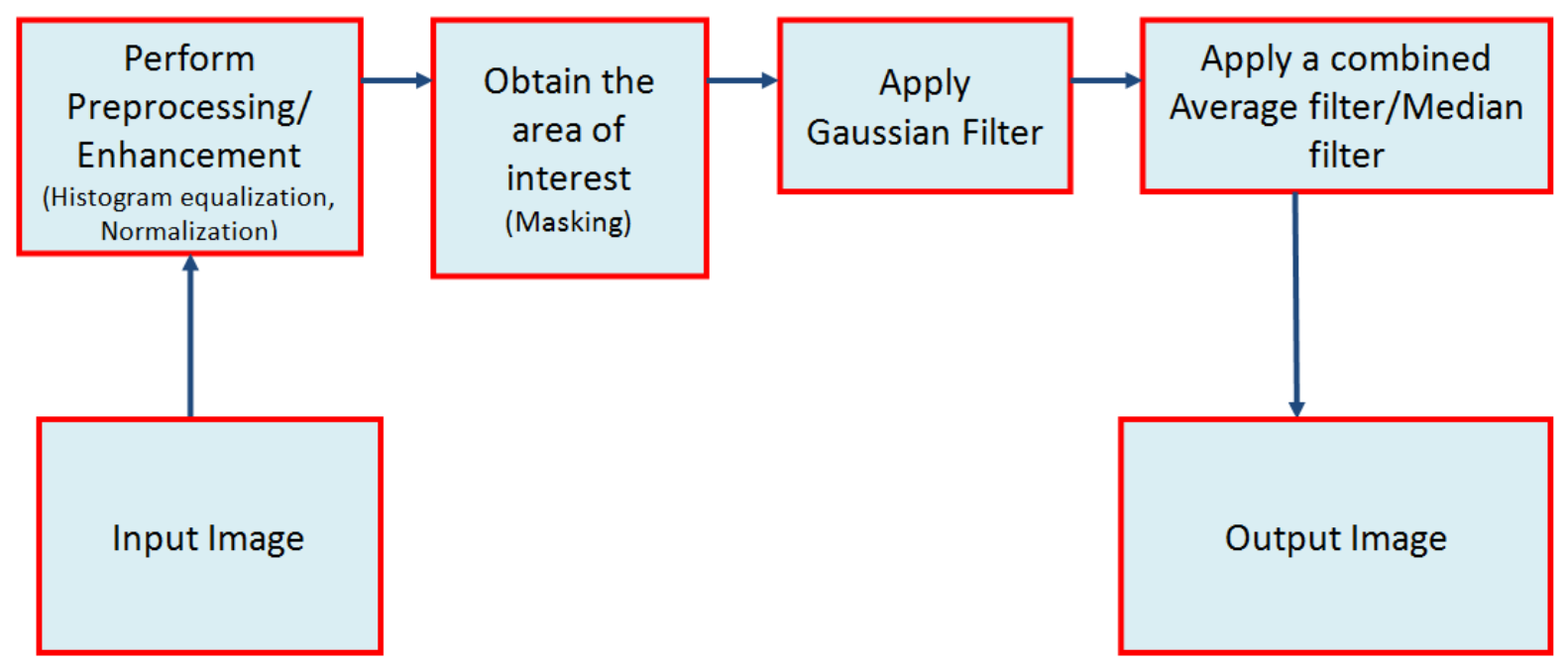

Figure 4.1: Flowchart of the pixel intensity based algorithm to detect creases.

\subsubsection{Pre-processing}

The pixel intensity based method operates under the assumption that the pixel intensity distribution patterns of the related creases in a given fingerprint are significantly different from those related to the valleys. This assumption is based on two criteria, namely empirical visual observations made by a human expert, and a study of pixel intensity histograms from the regions containing creases.

It was observed that a significant number of the images in our database were not suitable for the proposed algorithm. Therefore, image enhancement techniques were used on the images to improve their quality. The main objectives of image enhancement are to improve the visual quality of the image and to make the image suitable for this particular task of crease detection. In the process of enhancement, the image pixel attributes are modified. Some of the well-known techniques used for image enhancement include thresholding transformation, grey level slicing, intensity normalization, and histogram equalization.

- Thresholding transformation is a process of transforming the image pixel intensities from an input image to an output image using a specific threshold. This results in an output image whose pixel intensities have a range of [0 1]. This operation can be mathematically expressed 
as:

$$
B(x, y)= \begin{cases}1 & \mathrm{~A}(x, y)>=\eta \\ 0 & \text { otherwise }\end{cases}
$$

where $\eta$ is the threshold, and $A(x, y)$ is the input image while $B(x, y)$ is the resulting output image.

- Grey level slicing is a widely used technique in many applications such as satellite imagery enhancements, monitoring water level and land surface changes. The main benefit of grey level slicing is that it emphasizes the intensities of grouped regions while diminishing the intensities of other ungrouped regions.

- Image intensity normalization is a method of spreading the range of pixel intensities of an image over the whole spectrum. This results in maximizing the span of grey scale variation. This process is also be referred as contrast stretching and is a widely used technique in digital signal processing. This process can be mathematically expressed as:

$$
B_{\text {new }}(x, y)=\frac{A(x, y)-\min (A)}{\max (A)-\min (A)}
$$

where $(x, y)$ are the row and column location of the pixel, $A$ is the original image and $B$ is the resulting image.

- Histogram equalization is a process of producing an image that has a uniform probability function. For example, an image that has a low contrast can be modified in such a way that the features that were not visible prior to histogram equalization become more visible to the human eyes. 
Based on the image domain in which the processing is performed, enhancement techniques can be classified into two major groups: frequency domain methods and spatial domain methods [16].

- Spatial domain methods refer to techniques that directly manipulate the pixel values in an image.

- Frequency domain methods manipulate the image in its Fourier transform, and invert them back to spatial domain using an inverse Fourier transform.

In this work, image intensity normalization is chosen as the first preprocessing step. From Figure 4.2(c), it can be observed that a normalized image appears visually better than an image obtained after histogram equalization. It was observed that histogram equalization resulted in black patches that constitute noise.



(a)

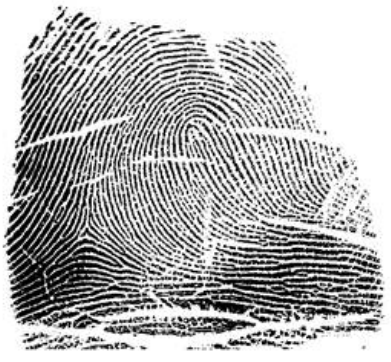

(b)

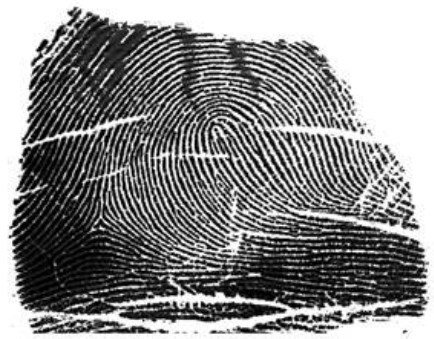

(c)

Figure 4.2: Effects of various preprocessing method: (a) Original image (b) image obtained after applying normalization (c) image obtained after applying histogram equalization.

\subsubsection{Obtaining the Region Of Interest (ROI)}

The region of interest in a fingerprint image is the surface area covered by the ridges, valleys and creases. The process therefore segments the fingerprint image from its background. A simple method of obtaining the region of interest is to determine the surface area of the fingerprint which has made contact with the sensor. This makes the image more suitable for crease detection as the 
area that contains the creases will be localized.

A quality measure of the fingerprint is also computed before identifying the region of interest. The output of this stage indicates whether or not the fingerprint is of good quality or not: prints with good quality are retained while those with bad quality are discarded. A method to obtain the quality measure is based on foreground area index [16]. In this method, the surface area of the fingerprint sensor that is assumed to be the foreground is measured. An image with a very high value of foreground area is regarded usable, while the images with low values are discarded.

The foreground area index value is computed using the following equation:

$$
I M_{q}=\frac{\sum_{F} I M(x, y)}{\sum_{\partial} I M(x, y)}
$$

where $(x, y)$ are the row and column location of the pixel, $F$ refers to the pixels where foreground information can be determined and $\partial$ refers to the total number of pixels in the area of interest. A high $I M_{q}$ represents a very good fingerprint. If $I M_{q}$ equals 1 , it suggest that the complete image is the foreground. Once the image quality has been determined, the region of interest is computed.

There are various methods for computing the region of interest. In this work we describe two methods that are used widely, keeping in mind that other methods that are not described in this work may provide equally good or better performance.

The first method is widely applied in many fingerprint enhancement algorithms. This method requires that the variable "block size" be specified. The block size affects the smoothness of the edges of the fingerprint in the region of interest. Standard deviation is applied on each block on the image and resulting values are obtained for each block. A threshold value is set to obtain the region of interest. Threshold value ranges between 0 and 1 . This algorithm is computationally less expensive when compared to other algorithms used for generating the region of interest. Experiments confirm that a large block size results in rough edges while a small block size produces smoother edges.

The second method for determining the region of interest is computationally more expensive. The major steps involved in the second algorithm are listed below.

(1) Image binarization: This operation yields an output in which the ridges appear in a highlighted black color, while valleys appear as white. 
(2) Ridge thinning: This process outputs an image in which the redundant pixels of ridges are eliminated, and the process is repeated until the width of the ridges is reduced to one pixel.

(3) Morphological operation: Image closing, hole filling and image erosion operations are applied to obtain the final area of interest.

This method produces a smooth-edged region of interest, however with a higher computational cost than the first method. Some sample images showing the ROI generation procedures using both the methods are seen in Figure 4.3.



(a)



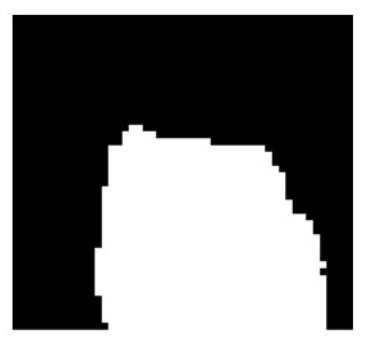

(b)

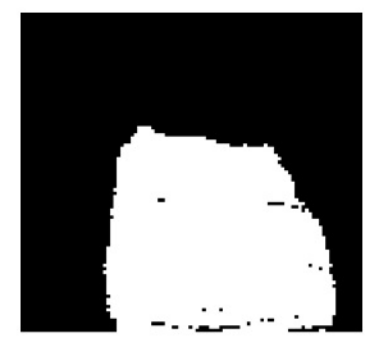

(c)

Figure 4.3: ROI generated using 2 different techniques (a) Original Image, (b) ROI with block size 16, (c) ROI with block size 8, (d) ROI with block size 2, (e) ROI using the second method.

Results from the different methods indicate that increasing the block size yields a better region of interest. However, the edges may not be completely smooth as seen in Figure 4.3(b). The area within the mask is completely white with the pixels having a value of 255 . Decreasing the block size to a value of 2 results in black patches within the region of interest, although the edges are smooth. For this work, the second method was used as it does not require specifying the block size and gives better output. Once the region of interest is computed, the next step is to superimpose the region of interest on the enhanced fingerprint images. Extracting creases based 
on the pixel intensity revolves around the visual identification of the creases. Therefore the area of the fingerprint covered by creases was visually localized, and some methods that isolate creases from valleys were applied.

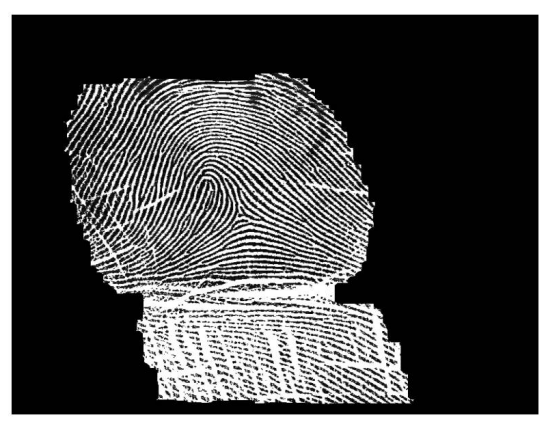

Figure 4.4: A mask superimposed on the enhanced image

To isolate the creases from the valleys, techniques such as Hough transform and edge and line mask were used initially. Some masks that were applied included horizontal and vertical masks as well , +45 and -45 degree masks. Some masks are shown in Figure 4.5. However, none of them yielded good performance. Therefore other robust methods based on mask filters were explored.

\begin{tabular}{|c|c|c|}
\hline-1 & -1 & -1 \\
\hline 2 & 2 & 2 \\
\hline-1 & -1 & -1 \\
\hline \multicolumn{3}{|l}{ Horizontal mask } \\
\hline
\end{tabular}

\begin{tabular}{|c|c|c|}
\hline-1 & -1 & 2 \\
\hline-1 & 2 & -1 \\
\hline 2 & -1 & -1 \\
\hline \multicolumn{3}{|c|}{+45 degrees } \\
\hline
\end{tabular}

\begin{tabular}{|c|c|c|}
\hline-1 & 2 & -1 \\
\hline-1 & 2 & -1 \\
\hline-1 & 2 & -1 \\
\hline \multicolumn{2}{|c|}{ Vertical mask } \\
\hline
\end{tabular}

\begin{tabular}{|c|c|c|}
\hline 2 & -1 & -1 \\
\hline-1 & 2 & -1 \\
\hline-1 & -1 & 2 \\
\hline \multicolumn{2}{|c|}{-45 degrees } \\
\hline
\end{tabular}

Figure 4.5: Different line mask filters

\subsubsection{Gaussian Filtering}

When Gaussian filtering was applied on the images a blurring effect was observed in the output images. This operation also helped in producing a noticeable contrast between the valleys and 
the creases. It was noticed that a pixel intensity based thresholding yields a better output. The assumption for thresholding based method is that if creases on images can be visually identified, then setting an optimal threshold value on pixel intensity would help in isolating the creases. Gaussian filter discriminates the creases from the valleys. However, setting a threshold did not completely isolate the creases from the valleys as some pixels in the valley were classified as creases and some creases were lost in the process.
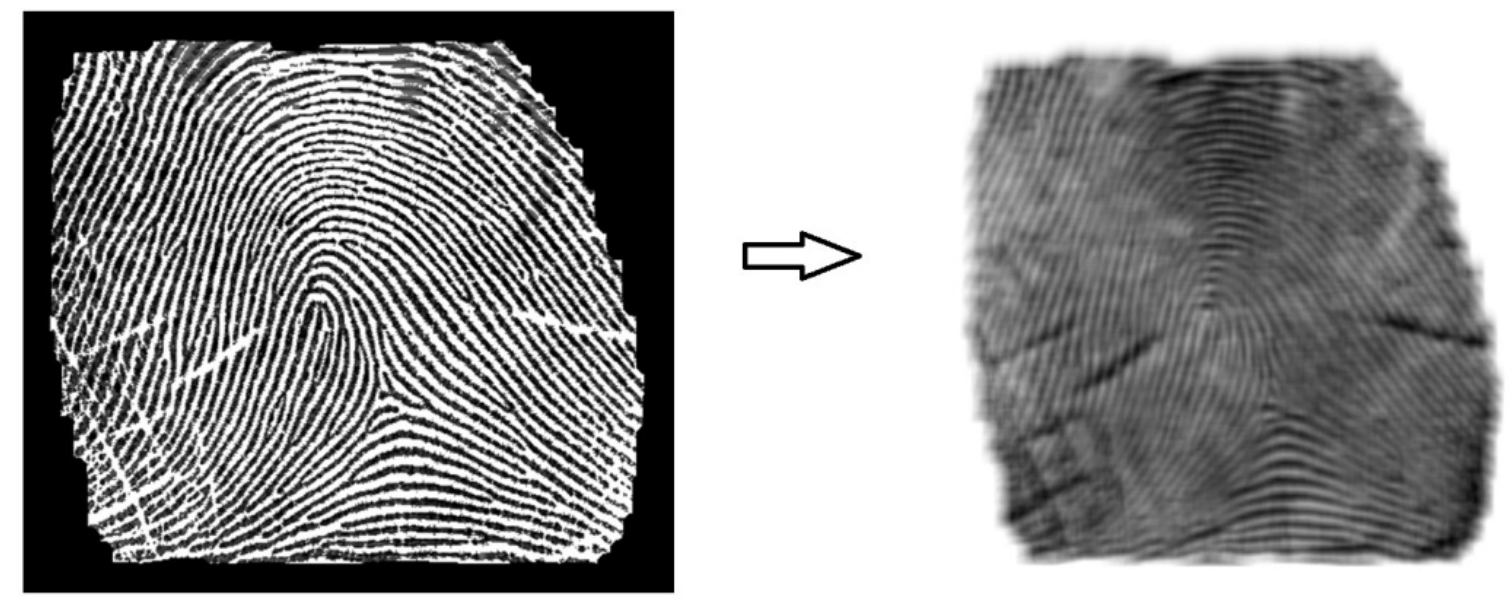

Figure 4.6: (a) Superimposed mask on the enhanced image (b) Image after applying Gaussian filter

It can be concluded that applying the Gaussian filter leaves the creases as dark black structures on the fingerprint image, as seen in Figure 4.6.

Determining a threshold that would optimally classify most creases may not be practically possible. Different threshold values were tested on the images in the database to determine a optimal value. The optimal threshold was empirically determined to be the mean value of the total image pixels divided by 2.1 . The pixel values lower than the threshold were classified as creases and were marked as black (value 0) while pixels with intensity values greater than the threshold were classified as non crease, and were marked as white (value 255).

From Figure 4.7, it can be noticed that the creases appear prominently after applying the threshold. However, some noise was still observed. This noise was observed to be from some valleys on the fingerprint with values above the threshold. Increasing the threshold value would remove most of the noise. However, some creases would be lost in the process. Decreasing the threshold would not reveal more creases, and would increase the noise in the image. 



Figure 4.7: (a) Image after applying Gaussian filter, and (b) after thresholding

\subsubsection{Post-processing}

The next step in the process was to implement a line detection algorithm on the image obtained after applying a Gaussian filter. The algorithm detects lines in the neighborhood of 2 pixels on the left and right, and 1 pixel on the top and bottom for each pixel on the image. For each point (origin) on the image, the algorithm checks if its neighbors has pixel intensity values greater than a particular threshold. If any of the neighbors have a pixel intensity value less than a threshold, it then calculates the mean of all its neighbors ( $4 \times 4$ block). If the mean is higher than the threshold, the particular point (origin) is retained, otherwise the point is discarded.

The benefit of this approach is that it removes most of the noise associated with the image. In Figure 4.7(b), the small sized patches on the image can be removed by applying the algorithm. The algorithm does not remove the creases because the width and length of creases do not fall into the category of small black patches based on the defined threshold.

The last step is to apply median filter on the image. Median filter smoothens the image, and makes the crease structures appear distinctly. The main challenge with the pixel intensity based method is the noise associated with each image. Other methods are still being explored to obtain more creases features. Figure 4.8 shows images obtained after post-processing was implemented on a few images. 

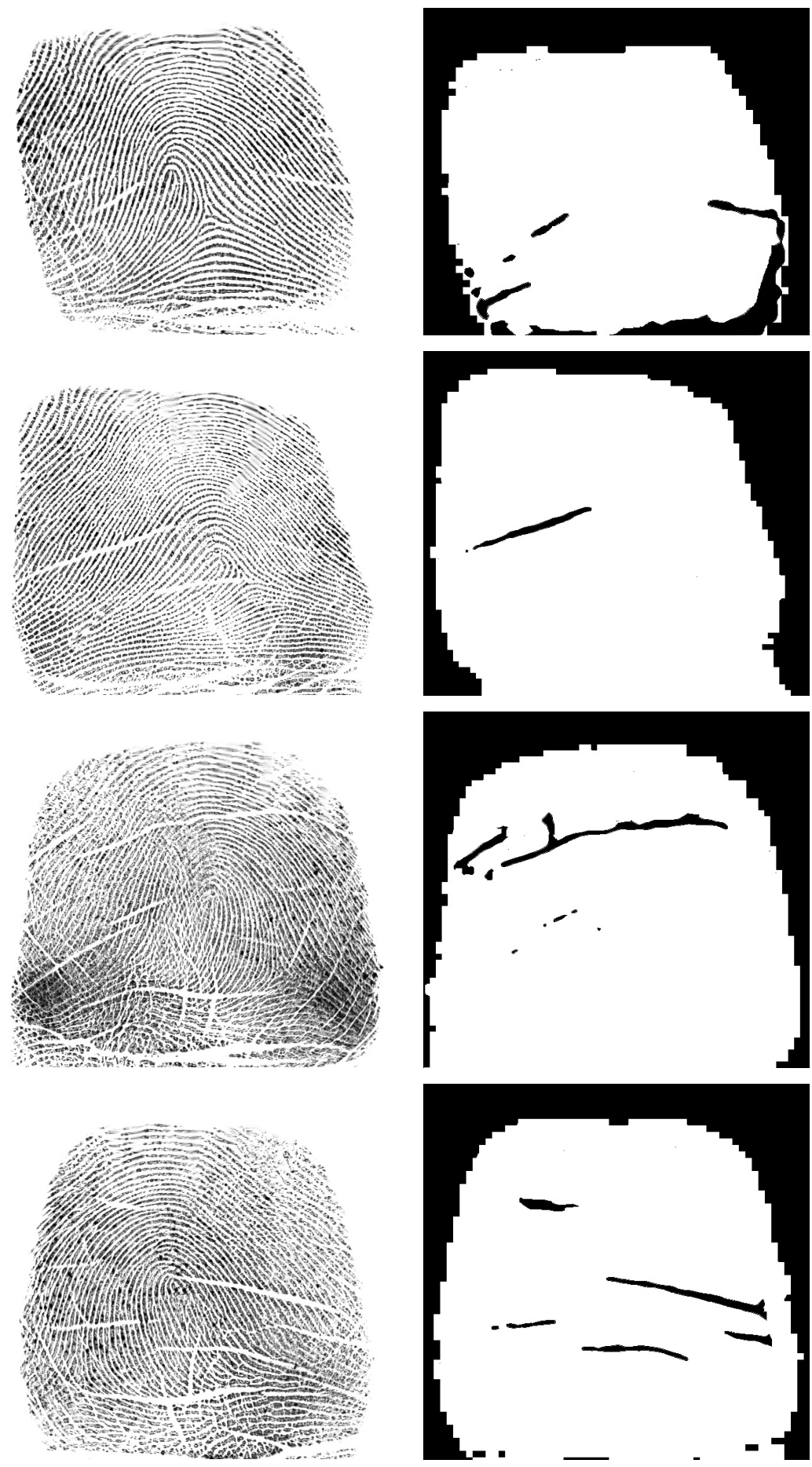

Figure 4.8: Sample output obtained after post-processing 


\subsection{Local ridge orientation field method}

Local ridge orientation field of a fingerprint has been found to be very useful in many applications including the detection of core and delta points in fingerprint images. The ridge orientation field describes the direction in which the ridges are positioned at each pixel.

The assumption behind the choice of this method is that the angular change in the ridge orientation field ceases to be consistent when a crease is encountered on the ridge path. The assumption holds true because the structure of creases will form lines that run across ridges and valley at a considerable angle, leading to early termination of such ridges. Therefore, obtaining ridge orientation gives a clue as to where the inconsistencies are located and hence suggest the presence of creases. It cannot, however, be concluded that all angular inconsistencies are caused due to presence of creases. Some inconsistencies are as a result of other fingerprint structures such as singular points. Singular points include the delta and core points.

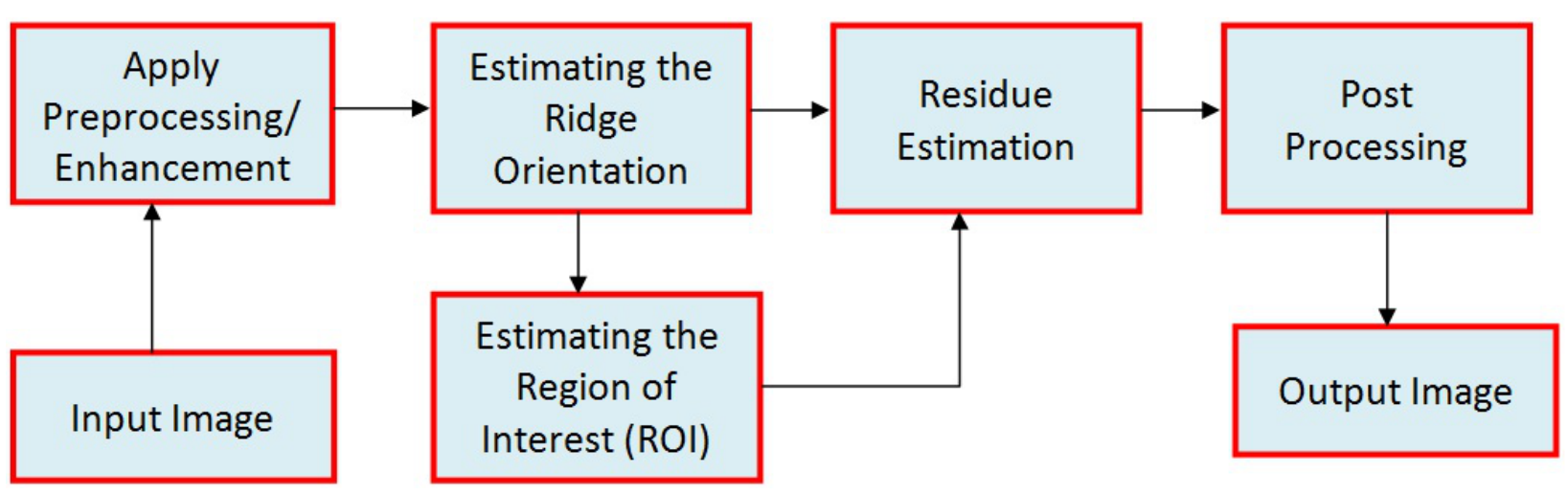

Figure 4.9: Schematic for crease detection based on ridge orientation field.

\subsubsection{Preprocessing/enhancement}

Due to the data collection process, which may not be carried out appropriately, some fingerprints may have poor image quality. Such images require some form of enhancement. The database used in this work requires image enhancement techniques to be applied, in order to make the images suitable for this work. These techniques have been discussed in section (3.1.1) of this thesis. The principal goal of enhancement is to improve the quality of the images to make them suitable for further processing. 


\subsubsection{Ridge orientation estimation}

Various techniques exist in literature that describe the process of orientation field estimation in fingerprint images. One of the popular technique is based on the square gradient averaging. This technique is used widely due to its simplicity.

\section{Gradient based approach:}

This method computes the gradients at each pixel, or discrete regions on the fingerprint [5]. Obtaining orientation fields at discrete positions is computationally less expensive because all the pixels are not processed. However, the orientation field obtained may not be the best. The algorithm for the gradient based approach can be described as follows:

- Given an image, each pixel has an associated $x$ value on the $\mathrm{x}$-axis and $y$ value on the $\mathrm{y}$-axis. These values are obtained. The image is then divided into non-overlapping square blocks.

- The orientation at each pixel is computed using the following equation:

$$
\begin{gathered}
\theta_{i j}=\frac{\pi}{2}+\frac{1}{2} \operatorname{atan} 2\left(2 G_{x x}, G_{x x}-G_{y y}\right), \\
\text { where } \\
G_{x x}=\sum_{h=-8}^{8} \sum_{k=-8}^{8} \nabla_{x}\left(x_{i}+h, y_{j}+k\right)^{2} \\
G_{y y}=\sum_{h=-8}^{8} \sum_{k=-8}^{8} \nabla_{y}\left(x_{i}+h, y_{j}+k\right)^{2} \\
G_{x y}=\sum_{h=-8}^{8} \sum_{k=-8}^{8} \nabla_{x}\left(x_{i}+h, y_{j}+k\right) . \nabla_{y}\left(x_{i}+h, y_{j}+k\right) \\
\nabla_{x} \text { and } \nabla_{y} \text { are the derivatives at } x \text { and } y \text { respectively. }
\end{gathered}
$$

The direction at each pixel represented by $\theta_{i j}$ has a range of [0 180] (see Figure 4.10). For each pixel in the image, a measure of reliability is computed. The reliability measure, represented as $r_{i j}$, describes the effect of noise on a particular pixel. A high $r_{i j}$ value represents high noise in the image while a low value of $r_{i j}$ represents less noise. The gradient based method, however, comes with some demerits. The quality of the orientation field may be adversely affected by the noise level in the image, which can cause false directions of the orientation field. 




Figure 4.10: Orientation field (gradient based) superimposed on a fingerprint.

Figure 4.11 depicts the effect of image quality on the fingerprint orientation field. This work assumes that the orientation field should have a consistent flow on the ridges, and that the points on the ridges that are discontinued due to the creases have an abrupt change in orientation. Therefore poor quality fingerprints are not preferred.

Figure 4.11(3) describes how an ideal image for this work looks like. The enlarged portion (top) describes the orientation flow which is consistent with the ridge flow and the second enlarged image (bottom) describes how the orientation responds to discontinued ridges. The next phase of our algorithm detects these points of discontinuity and labels them as potential crease locations.

- Matching based on orientation field

Holambe [19] attempted to match fingerprint based on orientation fields. Ridge orientation field can be used directly, or combined with fingerprint texture for matching [19],[20]. Holambe [19] computed the orientation fields using the gradient based method. The variance feature vector of the orientation field was also obtained. It was inferred that vector values were discriminative enough to be used for matching. The vector map can be computed using the equation:

$$
\sigma_{k}^{2}=\sum_{i=1}^{n}\left(\theta(i, k)-\mu_{k}\right)^{2}
$$

where $n$ and $m$ are the row and column count, $\mu_{k}$ is the mean of $k_{t h}$ column, and $\theta(i, k)$ is the $k t h$ column of $\theta(i, j)$. 


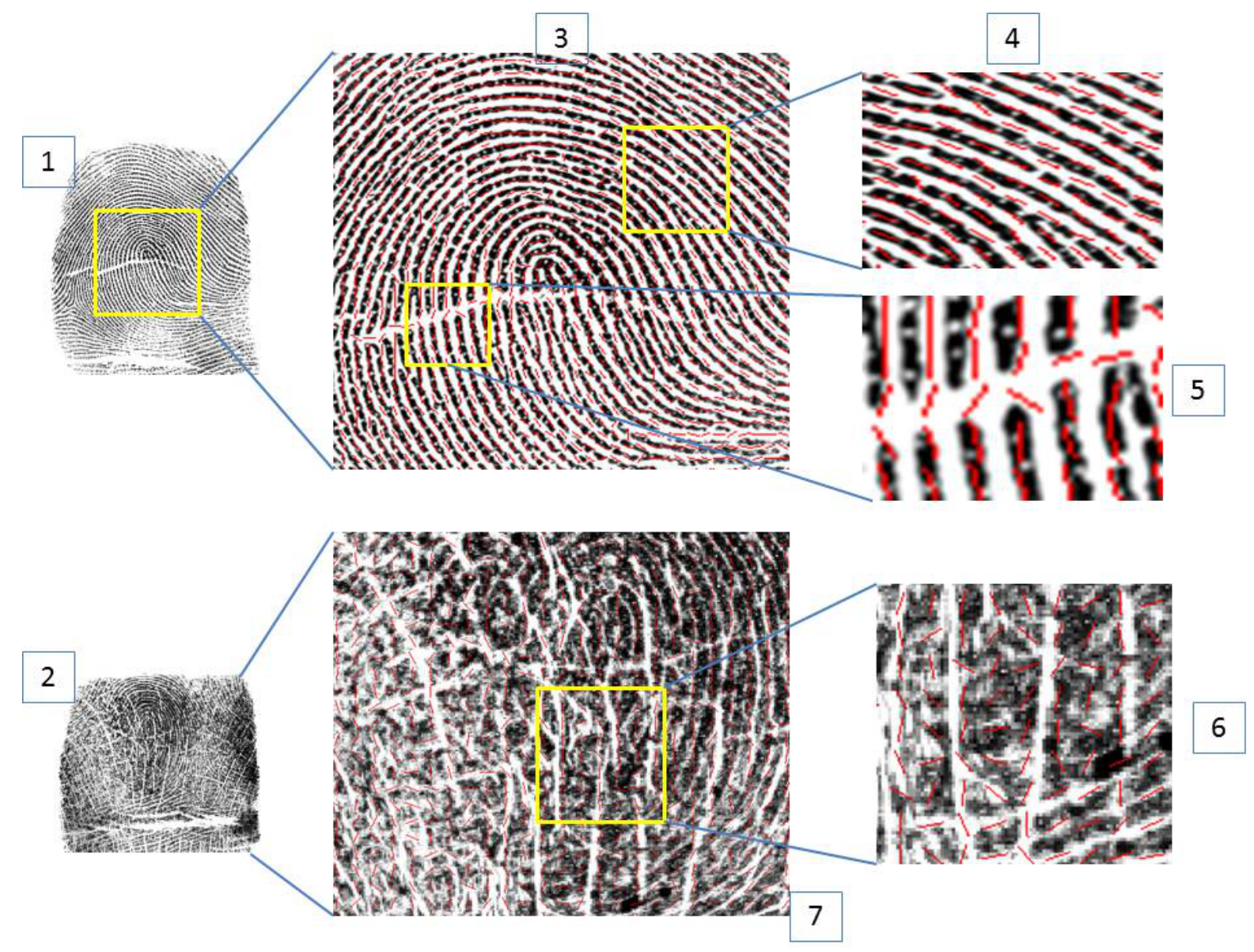

Figure 4.11: Effect of image quality on ridge orientation field (1) Good quality fingerprint (2) Poor quality fingerprint.

The resulting vector is the transpose of the collection of $\sigma_{k}^{2}$, defined by

$$
\bar{V}=\left[\sigma_{1}^{2}, \sigma_{2}^{2}, \sigma_{3}^{2}, \ldots \sigma_{n}^{2}\right]^{T}
$$

The vector obtained can be considered as the template used for matching. Matching is done based on distance measure between the vectors of two fingerprints and is computed using the following equation:

$$
d=\|\bar{V}-\bar{V}\|^{2}
$$

where $\bar{V}$ and $\bar{V}$ correspond to fingerprints from the query and gallery, respectively.

Sein [20], aligned images in the database, and the resulting orientation fields were computed. The effective intersection of regions in the two images to be matched were computed. Matching 
scores were computed based on the intersected regions using the equation:

$$
\operatorname{Score}(\operatorname{Img} A, \operatorname{Img} B)=\frac{1}{N} \Sigma_{i, j \in \tau} \delta(i, j)
$$

where $\delta(i, j)$ is the difference between the orientation points and $\tau$ is the collection of intersection points in the effective region. The difference between the orientation points was computed with the equation:

$$
\begin{gathered}
\delta(i, j)= \begin{cases}\delta_{0}(i, j) & \text { if } \delta_{0}(i, j)<\frac{\pi}{2} \\
\frac{\pi}{2}-\delta_{0}(i, j) & \text { otherwise }\end{cases} \\
\delta_{0}(i, j)=\left|\theta_{\text {imgA }}(i, j)-\theta_{\text {imgB }}(i, j)\right|
\end{gathered}
$$

where $\theta_{i m g A}(i, j)$ and $\theta_{i m g B}(i, j)$ are the orientations at $(\mathrm{i}, \mathrm{j})$ in the two images. A threshold is defined such that if $\operatorname{Score}(\operatorname{Img} A, \operatorname{Img} B)$ is greater than the threshold, it can be considered as a match between the two points and non-match otherwise.

As a part of the residue detection algorithm (that will be discussed later), there is a need to obtain the region of interest of the image. The region of interest and the ridge orientation field are required input to the residue detection algorithm.

\subsubsection{Region of interest estimate (ROI)}

The region of interest is the region within fingerprint images characterized by structures such as the ridges, valleys and creases. One of the important objectives of finding the region of interest is the reduction of computation cost, by considering the reduced image size.

\subsubsection{Residue Estimation}

Phase residue estimation is a process in which the parts of a given image are identified as residue points; residual points are the points that are located around ridge discontinuities or sharp spikes caused by a change in the ridge direction on fingerprints. Such points can be detected around the creases and singular points based on the phase information of a fingerprint. In this work, the phase information refers to the orientation field of the fingerprint. A technique based on the concept of 2D phase unwrapping was employed by Goldstein et al. [21] and Othman and 


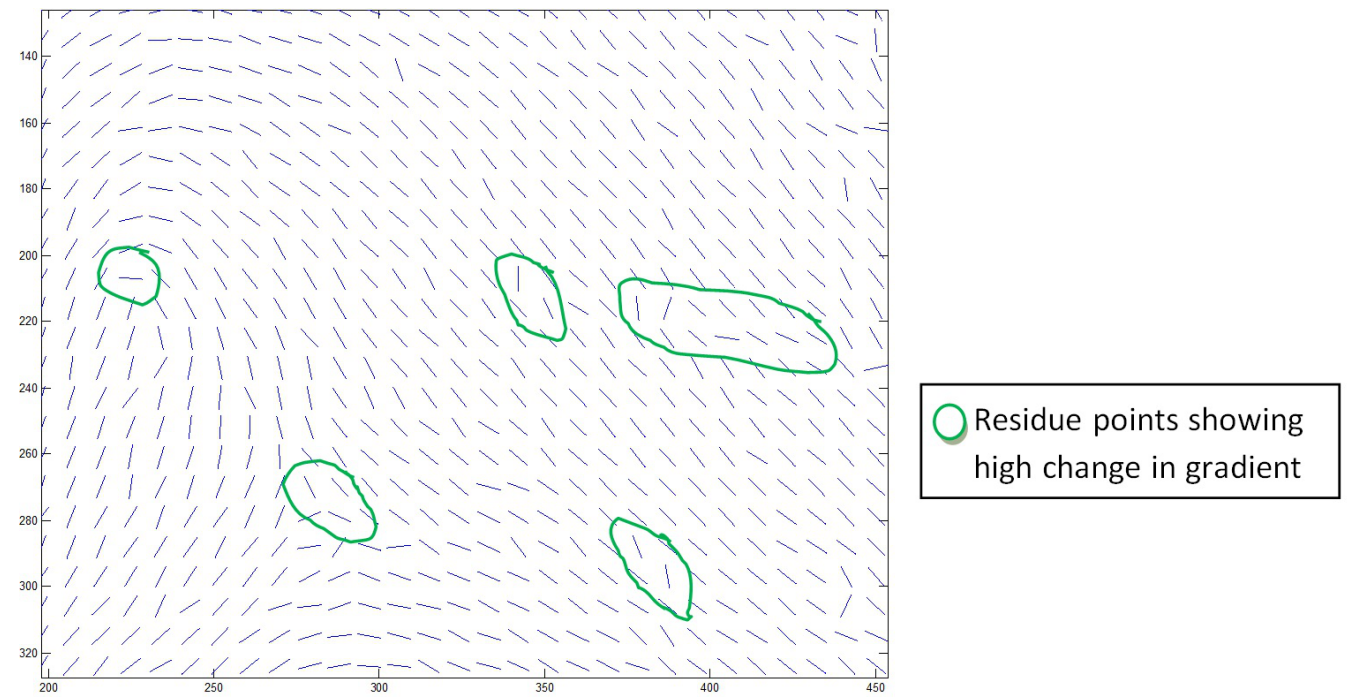

Figure 4.12: Ridge orientation field of a fingerprint showing regions containing residue points

Ross [30]. This technique is widely applied in satellite radar interferometry (SRI) of high velocity gas flows [21] and also in automated topography of land surface where discontinuities exist [22]. This concept has also been used to monitor the motion that occurs on ice sheets given an interval of few days. The principle behind the phase unwrapping was discussed by Goldstein and the idea is to identify portions and signal phases in a radar image where there exist unrelated amplitude and phase values which are referred to as signal noise or data discontinuities. A residue detection algorithm is then applied to identify the locations of such data discontinuities. The discontinuities are computed by the phase change or the gradient between every two points in the image.

\subsubsection{Post Processing}

It is still necessary to remove the noise left in the image, to ensure that we have images with only creases. Various methods were explored and the Euclidean distance between detected crease pixels gave the optimal result in removing most of the noise. The choice of Euclidean distance is because the points on the creases are tightly packed and clustered, and points that are sparsely distributed are suspicious points that constitute noise and hence need to be removed. Thresholding was applied to the Euclidean distance computed between two points to distinguish noisy pixels from crease pixels. 


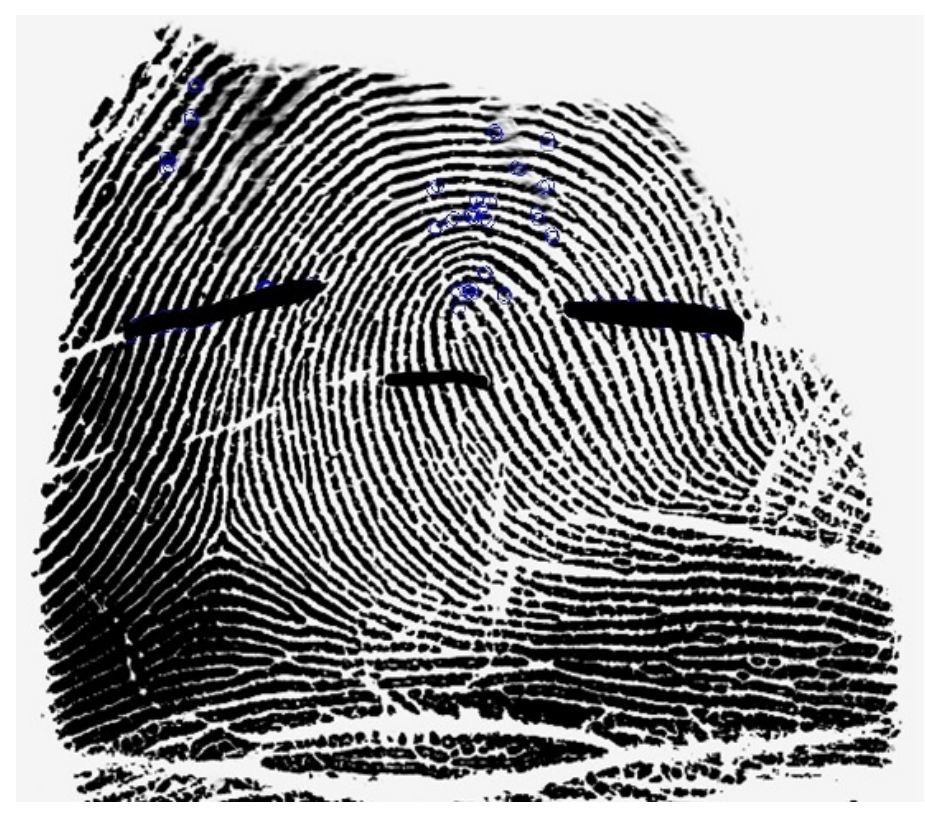

Figure 4.13: Fingerprint with the creases after applying Euclidean distance at a given threshold. 


\subsection{Validation of automated crease detection algorithm}

Two algorithms were designed in this work to detect the presence of creases on fingerprints. They are based on the two approaches described in the first two sections in this chapter. Method(1) is based on pixel intensity while method(2) is based on locations of residue points on fingerprint images.

Experiments were performed on a database made up of 131 samples. Samples were randomly selected from a larger pool of fingerprints. All the samples have creases on them with some having very visible creases while some had creases that were less discernible. The choice of having a database with varying crease visibility extent was to determine how robust the algorithms performed in the presence of poor quality fingerprints.

Method(1) which is based on pixel intensity gave good performance on fingerprint images with creases that were very visible, and whose intensity values were in contrast to that of valleys. Sample results from the algorithm are shown in Figure 4.14.

It can also be observed that the creases have wider width compared to the valleys, and the creases
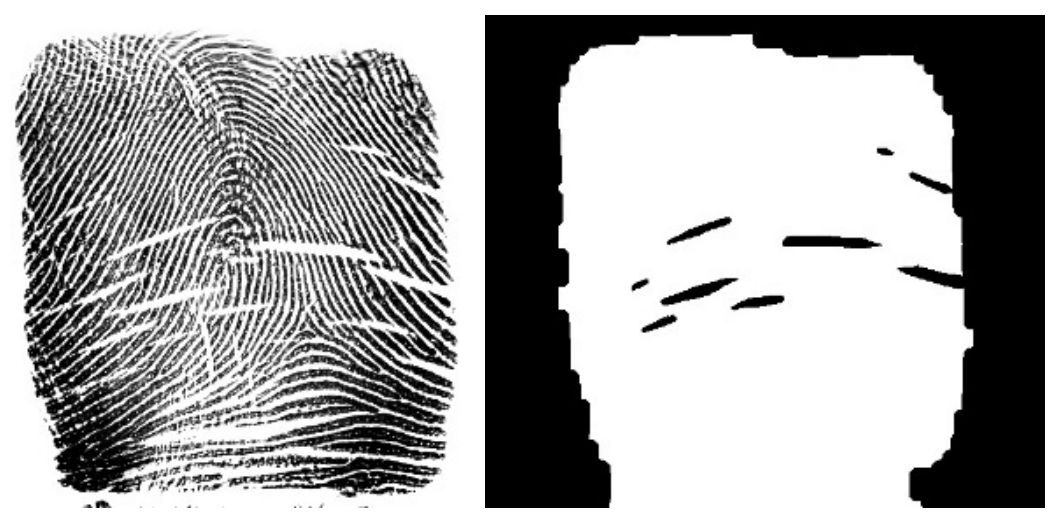

Figure 4.14: Results obtained using method(1).

also appear much visible than other features on the fingerprint.

Method(2) which is based on the residue points located on a fingerprint image performed well on fingerprints that had ridge terminations mostly along the creases. There were a few fingerprints in the database that had scattered and widely spread terminations on the entire fingerprint and method(2) did not perform well on such images. Figure 4.15 describes a fingerprint with residue points located mostly along the creases. 



Figure 4.15: Performance of crease detection on a fingerprint image using method(2).

Validation was also performed quantitatively based on the results of the two methods presented. The major steps involved in the quantitative validation are listed below:

- Three copies of the database were created, each made up of 131 samples. The first had the manually marked creases while the second and third corresponded to the application of the automated crease detection techniques based on method(1) and method(2).

- The creases in all the images in the first database were manually marked and the pixels were counted automatically using MATLAB

- The algorithm for method(1) was run on all images in the second database, and the creases detected were confirmed to be aligned with the original creases in the first database that were marked. All crease points that were aligned were counted automatically using MATLAB.

- The algorithm for method(2) was run on all the images in third database, and creases detected were confirmed to be aligned with the original creases in the first database that were marked. All points that were aligned were counted automatically using MATLAB.

- Tables were generated based on the above steps and were analyzed. Table 4.1 is a sample table generated based on step 2. Table generated from step 3 is also similar to Table 4.1.

The tables generated in step 4 were analyzed and performance of both methods were reported. The performance of both methods were about the same in terms of percentage of creases automatically detected when compared to the manually marked creases in the first database. Table 4.2 
Table 4.1: Data describing the percentage of crease detected based on method(1)

\begin{tabular}{|c|c|c|c|}
\hline Sample number & $\begin{array}{l}\text { Pixel count (man- } \\
\text { ually marked) }\end{array}$ & $\begin{array}{l}\text { Normalized } \\
\text { Pixel count } \\
\text { (Automated) }\end{array}$ & $\begin{array}{l}\text { True detection } \\
\text { rate (creases) }\end{array}$ \\
\hline 40 & 1810 & 1759 & $97.21 \%$ \\
\hline 2 & 6902 & 6515 & $94.40 \%$ \\
\hline 26 & 1621 & 1519 & $97.73 \%$ \\
\hline 1 & 6521 & 5286 & $81.06 \%$ \\
\hline 36 & 3415 & 2421 & $70.91 \%$ \\
\hline 10 & 2886 & 1992 & $69.03 \%$ \\
\hline 124 & 2397 & 1637 & $68.29 \%$ \\
\hline 6 & 4888 & 3333 & $68.20 \%$ \\
\hline 16 & 3752 & 2445 & $65.18 \%$ \\
\hline 38 & 3949 & 2534 & $64.18 \%$ \\
\hline 45 & 1615 & 967 & $59.92 \%$ \\
\hline 37 & 3026 & 1679 & $55.50 \%$ \\
\hline 3 & 6603 & 3423 & $51.84 \%$ \\
\hline 76 & 2104 & 1069 & $50.80 \%$ \\
\hline 100 & 7821 & 3881 & $49.69 \%$ \\
\hline 122 & 6448 & 3144 & $48.76 \%$ \\
\hline 5 & 5668 & 2725 & $48.09 \%$ \\
\hline 19 & 2335 & 1115 & $47.79 \%$ \\
\hline 95 & 1554 & 740 & $47.65 \%$ \\
\hline 129 & 4957 & 2275 & $45.90 \%$ \\
\hline 22 & 6326 & 2892 & $45.71 \%$ \\
\hline .. & .. &.$\cdot$ & .. \\
\hline.. & .. &.. & .. \\
\hline
\end{tabular}


Table 4.2: Performance analysis from method(1) based on pixel intensity

\begin{tabular}{|l|l|}
\hline Percentage of area detected & Number of samples \\
\hline $90 \%-100 \%$ & 3 \\
\hline $80 \%-89.9 \%$ & 1 \\
\hline $70 \%-79.9 \%$ & 1 \\
\hline $60 \%-69.9 \%$ & 5 \\
\hline $50 \%-59.9 \%$ & 4 \\
\hline $40 \%-49.9 \%$ & 15 \\
\hline $30 \%-39.9 \%$ & 27 \\
\hline $20 \%-29.9 \%$ & 31 \\
\hline $10 \%-19.9 \%$ & 25 \\
\hline $0.0 \%-9.9 \%$ & 19 \\
\hline
\end{tabular}

describes the breakdown of performance based on the creases detected by method(1) while Table 4.3 represents the performance for $\operatorname{method}(2)$.

Table 4.2 describes the performance of crease detection based on pixel intensity. The left column is the percentage of detected creases while the right column is the number of samples that fall into each of the groups. The algorithm detects over $90 \%$ of creases in 3 samples in the database while 19 samples had only $0-9.9 \%$ of their creases detected. Some images had creases and valleys that had about the same width and also same pixel intensity; hence, creases on such images were not detected accurately.

Figure 4.3 describes how the residue point estimation algorithm performs over the database of 131 images. The performance was not as good as the pixel based method. Detection rate of over $90 \%$ occurred in 1 sample while 30 samples had detection rate less than $10 \%$.

Based on the results in Figures 4.1, graphs were generated and are presented in Figure 4.16. Similarly, based on the results from method(2), graphs were generated and presented in Figure 4.17.

The graphs report the performance of the methods in this work. Further work is certainly necessary to improve crease detection performance. 
Table 4.3: Performance analysis from method(2) based on residue points

\begin{tabular}{|l|l|}
\hline Percentage of area detected & Number of samples \\
\hline $90 \%-100 \%$ & 1 \\
\hline $80 \%-89.9 \%$ & 1 \\
\hline $70 \%-79.9 \%$ & 9 \\
\hline $60 \%-69.9 \%$ & 7 \\
\hline $50 \%-59.9 \%$ & 12 \\
\hline $40 \%-49.9 \%$ & 10 \\
\hline $30 \%-39.9 \%$ & 15 \\
\hline $20 \%-29.9 \%$ & 23 \\
\hline $10 \%-19.9 \%$ & 23 \\
\hline $0.0 \%-9.9 \%$ & 30 \\
\hline
\end{tabular}

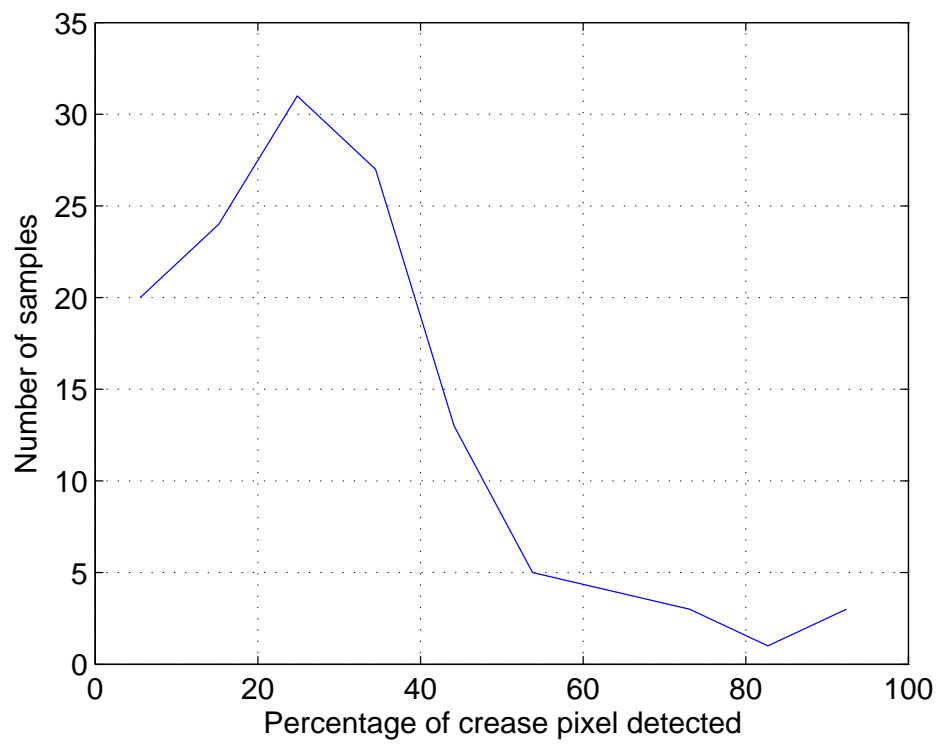

Figure 4.16: Graph displaying performance of method(1). 


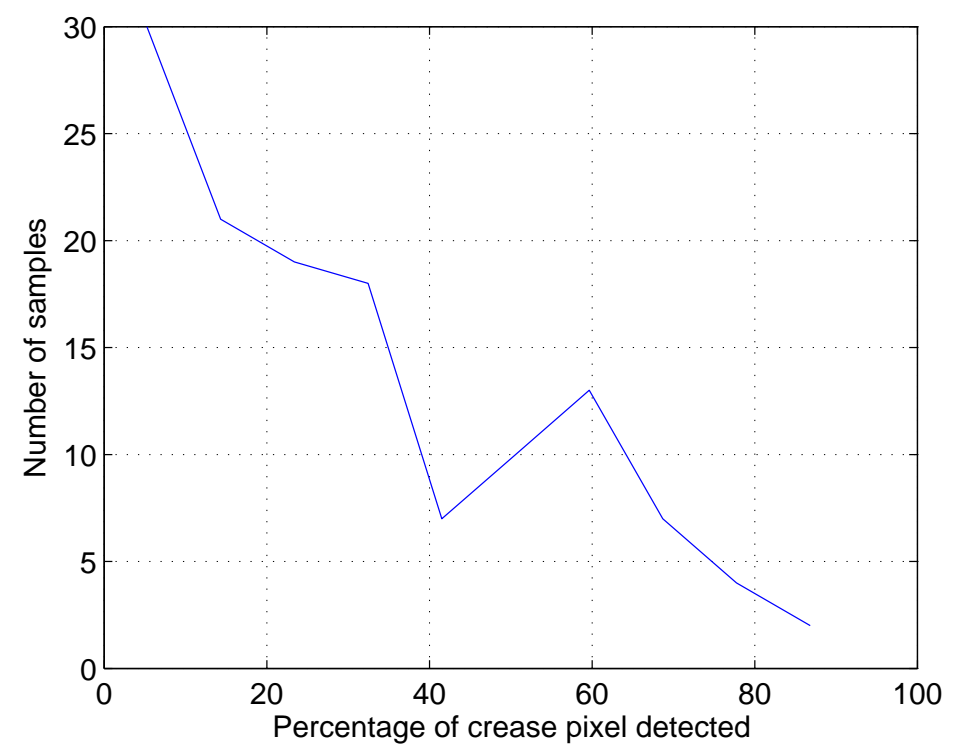

Figure 4.17: Graph displaying performance of method(2). 


\section{Chapter 5}

\section{Conclusion}

This thesis has provided insight into (1) the use of creases in fingerprint matching; (2) relationship between creases and age groups; (3) some methods to automatically detect creases. Matching performance based on manually detected creases indicates its potential usefulness in recognition. Evaluation based on equal error rate indicates a performance as low as $8 \%$ on a database of 101 fingers. Experimental results also suggest that performance increases when crease lines are sampled at frequent intervals; however this comes at a high computation cost in generating matching scores. There seems to exist a relationship between the creases on the fingerprint and different age groups (for those subjects having creases). Results also suggest that older people in the age range of 50 - 80 years are more likely to have creases covering a larger area on the fingerprint than those in the age group of 0 - 20 years. Automatic crease detection in fingerprint is not trivial. Two methods were considered in this work. The first method is based on pixel intensity and the second is based on identifying the residue points on a fingerprint ridge orientation map. Performance of the methods is dictated by the quality of the fingerprint. It can be concluded that fingerprints with visibly identifiable creases gave good results when applying the first method based on pixel intensity. The second method based on fingerprint orientation field scaled well on fingerprints that had high orientation field disparity along the creases.

Fingerprint creases can also be detected in low resolution images while minutiae point detection may give poor results in low resolution images. A consequence of this is the possibility of combining minutiae-matching scores with crease-matching scores in low resolution images. Fingerprint creases can also provide soft biometric information that can be used for fingerprint 
classification.

This work is exploratory and it points to a few areas of research in the future. It would be interesting to confirm if other parameters extracted from the creases can be used for gender and age classification. It would also be desirable to determine other sophisticated crease detection models. 


\section{References}

[1] A. Jain, A. Ross, and S. Prabhakar, "An Introduction to Biometric Recognition", IEEE Transactions on Circuit and Systems for Video Technology Volume 14, No. 1, January 2004

[2] S. Kimura, and B. Schaumann, "Embryological development and prevalence of thumb flexion creases", The Anatomical Record, Volume 222, Issue 1, pp 8389, September 1988.

[3] M.D. Femina, and A.A. Irudhayaraj, "Biometric System",Electronics Computer Technology (ICECT), 2011 3rd International Conference, Volume 1, pp 152-156, 2011.

[4] G.L. Wright, “Thumbs up for autocorrelation”, Security and Detection, European Convention, pp. $252-256,1995$.

[5] D. Maltoni, D. Maio, A. K. Jain, and S. Prabhakar, Handbook of fingerprint recognition, 1st ed. New York, Berlin Heidelberg: Springer-Verlag, 2003.

[6] A. Ross, A. Jain, S. Pankanti, "Biometrics: A Tool for Information Security",Information Forensics and Security, vol. 1, no. 2, pp. 125 - 143, June 2006.

[7] W.J. Babler, "Quantitative Differences in Morphogenesis of Human Epidermal Ridges”, Birth Defects Original Article Series. 1979; Vol. 15(6) pp 199-208, 1979.

[8] W.J. Babler, "Embryologic Development of Epidermal Ridges and Their Configurations", Birth Defects Original Article Series, Volume 27, Number 2, pp. 95 - 112, 1991.

[9] D. Gurdoglanyan, "Fingerprints used in Forensic Investigations,"[online] available at http://www.bxscience.edu/publications/forensics/articles/fingerprinting/r-fing01.htm. 2001.

[10] J. Zhou, F. Chen, N. Wu, and C. Wu, "Crease detection from fingerprint images and its applications in elderly people,'Pattern Recognition, 42, pp 896 - 906, 2009.

[11] J. Zhou, C. Wu, Z. Bian, and G. Rong, "Robust crease detection in fingerprint images", Proceedings of the 2003 IEEE Computer Society Conference on Computer Vision and Pattern Recognition, 2003.

[12] Hough Transform, [online], Available at http://en.wikipedia.org/wiki/Houghtransform.

[13] G. Shahan, "Heredity in Fingerprints", International Association for Identification, Vol 20, No. 4, April 1970. 
[14] J. Buzby, "Age vs. Cell Division Rate", [online] available at http://www.newton.dep.anl.gov/askasci/mole00/mole00313.htm.

[15] Boston children hospital, [online] available at www.childrenshospital.org/az/Site1073/mainpage.html.

[16] P. Hymer, "Extraction and application of secondary crease information in fingerprint recognition systems”, Master's Thesis, Institutionen fr teknik och naturvetenskap, March 2005.

[17] R. Maini and H. Aggarwal, "A Comprehensive Review of Image Enhancement Techniques". Journal of Computing, Vol 2, Issue 3, March 2010,

[18] W. Yang, X. Xu, K. Bi, S. Zeng, Q. Liu and S. Chen, "Adaptive region of interest method for analytical microCT reconstruction”, Journal of Xray Science and Technology 19, pp. 23-33, 2011.

[19] J. Kulkarni, B. Patil, R. Holambe, "Orientation feature for fingerprint matching", Pattern Recognition 39 pp. 1551 - 1554, 2006.

[20] Z. Win, M. Sein, "Robust Fingerprint Recognition System using Orientation and Texture features", 2nd International Conference on Computer Science and Information Technology (ICCSIT'2012) Singapore, April 28-29, 2012.

[21] R. Goldstein, H. Zebker,and C. Werner, "Satellite radar interferometry: two dimensional phase unwrapping", Radio Science, Number 4, pp.713-720, August 1988.

[22] D. Bone, "Fourier fringe analysis: the two-dimensional phase unwrapping problem", Applied Optics, vol. 30, no. 25, September 1991.

[23] H. Zebker, Y. Lu, "Phase unwrapping algorithms for radar interferometry: residue-cut, leastsquares and synthesis algorithm", J. Opt. Soc. Am. A/Vol. 15 No. 3/ March 1998.

[24] A. Badawi, M. Mahfouz, R. Tadross, R. Jantz, "Fingerprint-based gender classification", [unpublised paper], 2008.

[25] G. Amayeh, G. Bebis, and M. Nicolescu, "Gender classification from hand shape". Computer Vision and Pattern Recognition Workshops, June 2008.

[26] Polydactyly, [online], Available at http://en.wikipedia.org/wiki/Polydactyly.

[27] Skin layer, [online], Available at http://gleeson9science.pbworks.com/w/page/27028466/Touch.

[28] A. Bazen, G. Verwaaijen, S. Gerez, L. Veelenturf and B. Zwaag, "A Correlation-Based Fingerprint Verification System”, ProRISC 2000 Workshop on Circuits, Systems and Signal Processing, Veldhoven, The Netherlands November 2000.

[29] B. Handwerk, Born Without Fingerprints, [online], http://news.nationalgeographic.com/news/2006/09/060922-fingerprints.html

[30] A. Othman, and A. Ross, "Mixing Fingerprints For Generating Virtual Identities", Proc. of IEEE International Workshop on Information Forensics and Security, December 2011. 